\title{
LA SENTENCIA SOBRE EL TERREMOTO DEL 6 DE ABRIL DE 2009 EN L'AQUILA, ITALIA: LECCIONES PARA LA GESTIÓN DEL RIESGO EN AMÉRICA CENTRAL
}

\author{
SENTENCE IN THE CASE OF THE APRIL 6, 2009 L'AQUILA, ITALY \\ EARTHQUAKE: LESSONS FOR RISK MANAGEMENT IN CENTRAL AMERICA
}

\author{
Sergio Mora \\ ELM-Consultorías; Humberto 1, 844, PB-A, Buenos Aires, Argentina \\ sergio.moracastro@gmail.com
}

(Recibido: 9/03/2014 ; aceptado: 13/05/2014)

\begin{abstract}
October 2008 to April 2009: A seismic swarm occurred in Abruzzo, Italy. March $30^{\text {th }}: \mathrm{A} \mathrm{M}_{\mathrm{w}} 4.0$ event induced L'Aquila's Mayor to evacuate public buildings; the population became alarmed. March $31^{\text {st: }}$ The Italian government convened the Commission of Major Risks to meet with local authorities. The Spokesman informed the public that “...there is no reason to believe the swarm as precursor to a major event...". April $6^{\text {th }}: \mathrm{A} \mathrm{M}_{\mathrm{W}} 6.3$ earthquake caused 309 fatalities, 1,500 injured, and 67,000 people lost their dwellings; buildings at the historic centre, student residences and a hospital were destroyed. In parallel, a technician without scientific background measured ground Radon gas emissions and published a correlation indicating a future earthquake. Even if in this case he "succeeded" in his forecast, the press forgot all his past failures. Six experts and the Deputy Head of Civil Protection were accused of manslaughter for having, among other charges, performed an approximate, generic and inefficient risk assessment, delivered with imprecise and ambiguous information, and by saying that data did not allow to conclude that the swarm came prior to a major earthquake. This trial could become a precedent leading to investing infinite resources in the prediction of events that cannot be predicted rather than emphasizing in shared responsibility for risk management: high level advisory committees, authorities, civil protection, press, scientists, population, building contractors and judges.

Keywords: Risk management, earthquake prediction, seismic hazard, prognosis, L'Aquila, risk communication, Italy.

RESUMEN: De octubre 2008 a abril 2009: Se produjo un enjambre sísmico en Abruzzo, Italia. 30 de marzo: Un sismo de $\mathrm{M}_{\mathrm{w}}$ 4,0 indujo al Alcalde de L'Aquila a evacuar edificios públicos; la población estaba nerviosa. 31 de marzo: El gobierno italiano convocó a la Comisión de Riesgos Mayores para reunirse con las autoridades locales; el vocero informó al público: “...no hay razón para pensar que el enjambre sea precursor de un evento mayor...". 6 de abril: Terremoto $\mathrm{M}_{\mathrm{w}} 6,3$; 309 muertos, 1.500 heridos, 67.000 personas perdieron sus viviendas; destrucción de edificios en el centro histórico, residencias estudiantiles y un hospital. Paralelamente, un técnico de laboratorio sin calificación científica, mediante la medición de emisiones de Radón en el terreno, publicó un indicador y su correlación con un sismo próximo. Aunque en este caso "acertó", la prensa olvidó todos sus desaciertos anteriores. Seis expertos y el subjefe de Protección Civil
\end{abstract}


fueron acusados de homicidio culposo, entre otros cargos por: Haber realizado una valoración aproximada y ambigua del riesgo y decir que los datos no permitían concluir que el enjambre fuese premonitor del terremoto. La sentencia es un precedente que podría desembocar en un proceso de inversión infinita de recursos para la predicción de eventos que no pueden predecirse, en lugar de enfocarse en la responsabilidad compartida de la gestión del riesgo: Comités de alto nivel, autoridades, protección civil, prensa, científicos, población, constructores y jueces.

Palabras clave: Gestión del riesgo, predicción sísmica, prognosis, amenaza sísmica, L’Aquila, comunicación del riesgo.

\section{INTRODUCCIÓN}

\section{Preámbulo y definiciones}

En este artículo se resume e interpreta la información sobre el caso del proceso judicial que se realiza en Italia acerca del terremoto de L'Aquila, sucedido el 6 de abril de 2009. El análisis, contenido y conclusiones no se realizaron bajo criterios estrictamente jurídicos y son solamente el producto de la interpretación de los datos, documentos e información accesibles y de carácter público, que ha sido posible de obtener mediante las bús-quedas y consultas en el Internet.

Con estas premisas conviene, para los propósitos de este artículo, comenzar por repasar algunos conceptos, términos y definiciones específicas y relevantes, los cuales serán tomados en cuenta en contexto del caso:

Pronóstico: De acuerdo con el Diccionario de la Real Academia Española (RAE, 2001) este vocablo pro-viene del latín prognosticum y del

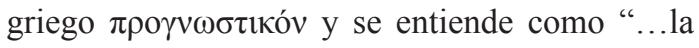
señal, por medio de la cual se conjetura o calcula un evento futuro...". En otras palabras, representa el juicio que formula un especialista con respecto a los eventos -sus causas, su curso y los cambiosque pueden sobrevenir, su duración y conclusión, todos interpretados de acuerdo con los síntomas que los preceden o acompañan. Se trata de conocer, por antici-pado, lo que sucederá en el futuro, descifrando la situación por aproximación y a través de indicios interpretables. Esta es, evidentemente, una actividad multidisciplinaria, en donde para que tengan sentido sus productos, debe con- verger el trabajo de un equipo diverso y complementario (National Commission for Forecasting and Preventing Major Risks, 2009).

Predicción: Según la RAE (2001), esta palabra se origina del vocablo latino praedicťonis, es decir, la “....acción y efecto de predecir...”. En este caso, se interpreta como la manifestación de aquello que es probable que suceda en el futuro, basándose en análisis y consideraciones de juicio. Su definición posee, además, algunas semejanzas con la del pronóstico, pero no son sinónimos.

Predecir, según la RAE (2001), es “....anunciar por revelación, ciencia o conjetura algo que ha de suce-der...", pero en este caso mediante una declaración precisa de lo que ocurrirá bajo condiciones determinadas. Ahora bien, los procesos naturales que se producen súbitamente, cuando no se cuenta con datos previos suficientes o cuando no es posible interpretarlos certeramente por causa de las dificultades, deficiencia o insuficien-cia en el conocimiento de las leyes naturales que los gobiernan, no pueden ser predichos.

Para el caso de la predicción de los sismos y terremotos, por convención y para que una "predicción" sea considerada seria y responsable desde el punto de vista científico, Scholz et al. (1973), Allen (1976), Jackson (1996) y Geller et al. (1997) indicaron algunos de los requisitos que deberían cumplirse con un grado de exactitud lo más próximo posible al de la realidad: i) Tiempo (i.e. fecha, hora); ii) Localización (i.e. coordenadas, profundidad); iii) Magnitud; iv) Grado de confianza, medición estadística-probabilística de la in-certidumbre y del error estándar de los cálculos; v) Indicación de la probabilidad de que el 
evento suceda, más bien, como el producto de un proceso aleatorio o casual y que no tenga que ver con la predicción; y vi) Publicaciones "ex-ante" y "ex-post" de la predicción, en las que tanto el éxito como el fracaso de los cálculos, tengan el mismo grado de visibilidad.

En todo caso, téngase en cuenta la opinión del Dr. Charles F. Richter, uno de los padres de la Sismología moderna (en Geller et al. 1977): “... Journalists and the general public rush to any suggestion of earthquake prediction like hogs toward a full trough... [Prediction] provides a happy hunting ground for amateurs, cranks, and outright publicity-seeking fakers..." (Los periodistas y el público en general se abalanzan hacia cualquier sugerencia relacionada con la predicción de un terremoto, cual cerdos hacia su comedero... [La predicción] ofrece un terreno de cacería muy agradable para los aficionados, farsantes y buscadores a ultranza de la publicidad. Además expresó: “... only fools and charlatans predict earthquakes..." (Charles F. Richter; en: Houg, 2007, p. 253). Por su parte, Amoruso \& Crescentini (2012) manifiestan que "...predicting earthquakes is challenging and maybe possible in the future..." (La predicción de los terremotos podría ser posible en el futuro) [Traducciones del autor].

Prognosis: Para la RAE (2001) el origen del vocablo proviene del griego $\pi \rho \gamma_{\gamma} \omega \omega \iota \varsigma$ y consiste en “...el conocimiento general y anticipado de las condiciones que rigen la gestación y consecuencias de un suceso...". En el ámbito de las ciencias y de la ingeniería, se considera que la prognosis consiste en descifrar, más que el cuándo y el sitio exacto, el resultado esperado de un evento, su intensidad y la probabilidad de sus consecuencias, basándose para ello en la formulación de modelos y escenarios rigurosamente científicos y verificables, al menos "ex-post". Esta definición se adapta muy bien al análisis de la amenaza sísmica.

Prospectiva: Según la RAE, este término proviene del latín prospicere, mirar, y consiste de “...un conjunto de análisis y estudios realizados con el fin de explorar o de predecir el futuro, en una determinada materia...". Se trata, en otras palabras, de una metodología o técnica que se emplea para analizar y anticipar escenarios futuros posibles (i.e. "futurología") y cuyo objetivo es estimar su probabilidad de ocurrencia. Se aplica, supuestamente, con el objeto de orientar la toma de decisiones de manera racional en relación a escenarios eco-nómicos, comerciales, tecnológicos o políticos y puede ser rigurosamente científica o no.

Seudociencia (paraciencia): De acuerdo con el Diccionario Larousse (2009), se trata de “...un saber coherente y organizado como una ciencia, pero que carece de rigor...". Se refiere, entonces, a los productos de una afirmación, creencia o práctica que a pesar de presentarse como científica, no se basa en un método verificable ni válido, pues carece de una medida de su plausibilidad, del apoyo de resultados certificables científicamente o que simplemente no pueden ser evaluados de forma fiable, objetiva e independiente.

La seudociencia se fundamenta en profecías, adivinación, clarividencia, alquimia, mentalismo, mitos, religión, sincretismos y magia. Reúne un conjunto de suposiciones y ficciones que se refieren a situaciones hipotéticas acerca del futuro, sin fundamento racional o bajo premisas que, por lo general, dependen de la fe, mística o creencias y que, en general, no tienen sustento científico, o simplemente están sesgadas y manipuladas. En muchos casos este procedimiento también se utiliza para falsificar y hasta distorsionar datos e información pública, de manera alarmista y sensacionalista, con fines de control religioso, comercial y político, o para atraer la atención y aumentar las ventas de algún medio de comunicación (Fig. 1). Este tipo de estrategias ha sido ampliamente utilizado, por ejemplo, en la difusión de la información acerca del calentamiento global antropogénico (Mora, 2012).

\section{Anticipación de los eventos geodinámicos}

En la realidad y cuando los procesos naturales se producen progresiva y gradualmente y se pueden observar, medir y modelar gracias a la 
aplicación de recursos instrumentales, matemáticos e informáticos abundantes, profusos y rigurosos, es posible determinar, desde el punto de vista científico y con cierta aproximación, dónde, cuándo y de qué manera se materializarían de nuevo. No obstante, por ahora este caso se limita a pocos tipos de eventos asociados con la meteorología, las inundaciones, algunos tipos de erupción volcánica, la trayectoria de corto plazo de los ciclones tropicales y algunas clases de incendio forestal y deslizamientos.

La predicción y el pronóstico de corto y mediano plazo (días, semanas, meses, años) e incluso largo plazo (decenios, siglos), depende primariamente de la calidad, confiabilidad y disponibilidad de los datos, de los re-cursos informáticos accesibles, del grado de detalle, resolución y precisión de los datos históricos -y prehistóricos- e instrumentales (i.e. series temporales con decenas y hasta centenas de años). También es imprescindible disponer de capacidades y destrezas para aplicar, no solamente recetas de "caja negra", sino tener el control completo de los modelos físicos, estadísticos, matemáticos y estocásticos requeridos y que por lo general son complejos y casi siempre muy costosos de aplicar. Pero además y aunque todo lo anterior exista, no se podrá avanzar sin el conocimiento y dominio completos de las leyes naturales que rigen los procesos geodinámicos y es aquí en donde por lo general se encuentra el obstáculo mayor.

Por estas razones es que, bajo el estado actual del conocimiento y de las capacidades de la ciencia y de la tecnología, es imposible pronosticar y predecir los terremotos (Wyss, 1997). Pero además es innecesario. Lo anterior es una realidad, aunque se intente en ocasiones ofrecer versiones seudocientíficas y sofismas que inten-tan demostrar lo contrario (Univisión, 2013).

Ahora bien, sí se considera necesaria la prognosis de la amenaza sísmica y de ahí, la asignación de su prioridad correspondiente dentro del contexto integral de la gestión del riesgo. Este criterio cobra importancia particular en los países "en vías de desarrollo", en donde los recursos humanos y financieros son casi siempre limi-tados, por lo que debe optimizarse su utilización y evitar su desperdicio.
Lo más importante es la cuantificación exante y probabilística de la amenaza y luego, sobre todo, las me-didas pertinentes para la reducción de la vulnerabilidad de los elementos expuestos (Mora \& Alvarado, 2012a, 2012b). Esto se logra mediante el conocimiento, la comunicación adecuada del riesgo y su cuantificación, de acuerdo con sus características, causas, distribución espacio-temporal de los efectos, impactos y las consecuen-cias probables (Mora, 2009, 2010).

\section{La sismicidad y sus mitos}

Frecuentemente se escucha entre la gente, o se lee en los medios de comunicación colectiva, que una sucesión de numerosos eventos símicos con magnitudes bajas evita la acumulación de la energía sismogénica y que, por lo tanto, el próximo sismo grande podría ser menos intenso. Esta afirmación no tiene fundamento científico demostrable (Red Sismológica Nacional de Costa Rica, 2013) y con frecuencia, en el pasado más bien se ha verificado lo contrario. Lo que sí es cierto, más bien, es que para liberar progresivamente la energía de un terremoto, como el sucedido en L'Aquila con magnitud $\mathrm{M}_{\mathrm{w}} 6,3$, se requeriría de la suma de al menos 30 sismos con magnitud $\mathrm{M}_{\mathrm{w}}$ 5,3, o de 900 sismos $M_{w}$ 4,3 (RSN, 2013).

Por otra parte, la micro-sismicidad y los "enjambres de temblores" se han considerado, a menudo, como síntomas precursores de un evento importante próximo a suceder. Aunque eso fue lo que sucedió esta vez en L'Aquila (Hall, 2011), también se ha observado, en muchos casos, que este criterio está lejos de ser una ley natural automática. Además, no es ni frecuente ni omnipresente en los ambientes sismo-tectónicos conocidos (Greenemeir, 2011). En muchas regiones del mundo ha sucedido y suele suceder, más bien, que la mayoría de los enjambres sísmicos termina sin producirse un terremoto intenso y que muchos sismos de magnitud grande suceden sin el escenario previo de un enjambre de sismos menores.

El tema de las emisiones del gas Radón en el terreno y su correlación con un sismo próximo posible ha sido discutido en numerosos foros científicos, pero hasta la fecha no se ha alcanzado 


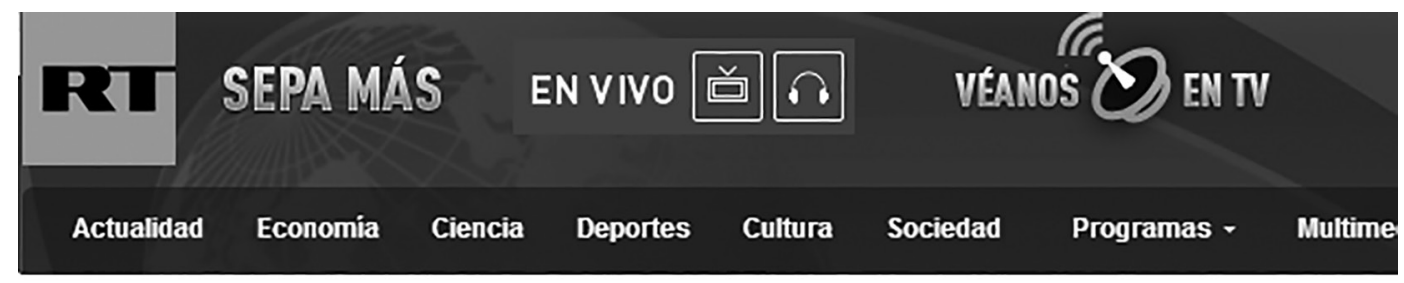

RT Actualidad/Actualidad

[a http:lles.rt.com/oXh Imprimir

\section{Científicos japoneses alertan de gigantesco terremoto "que está por venir" \\ Publicado: 31 may 2013 | 0:15 GMT Última actualización: 31 may 2013 | 16:22 GMT}

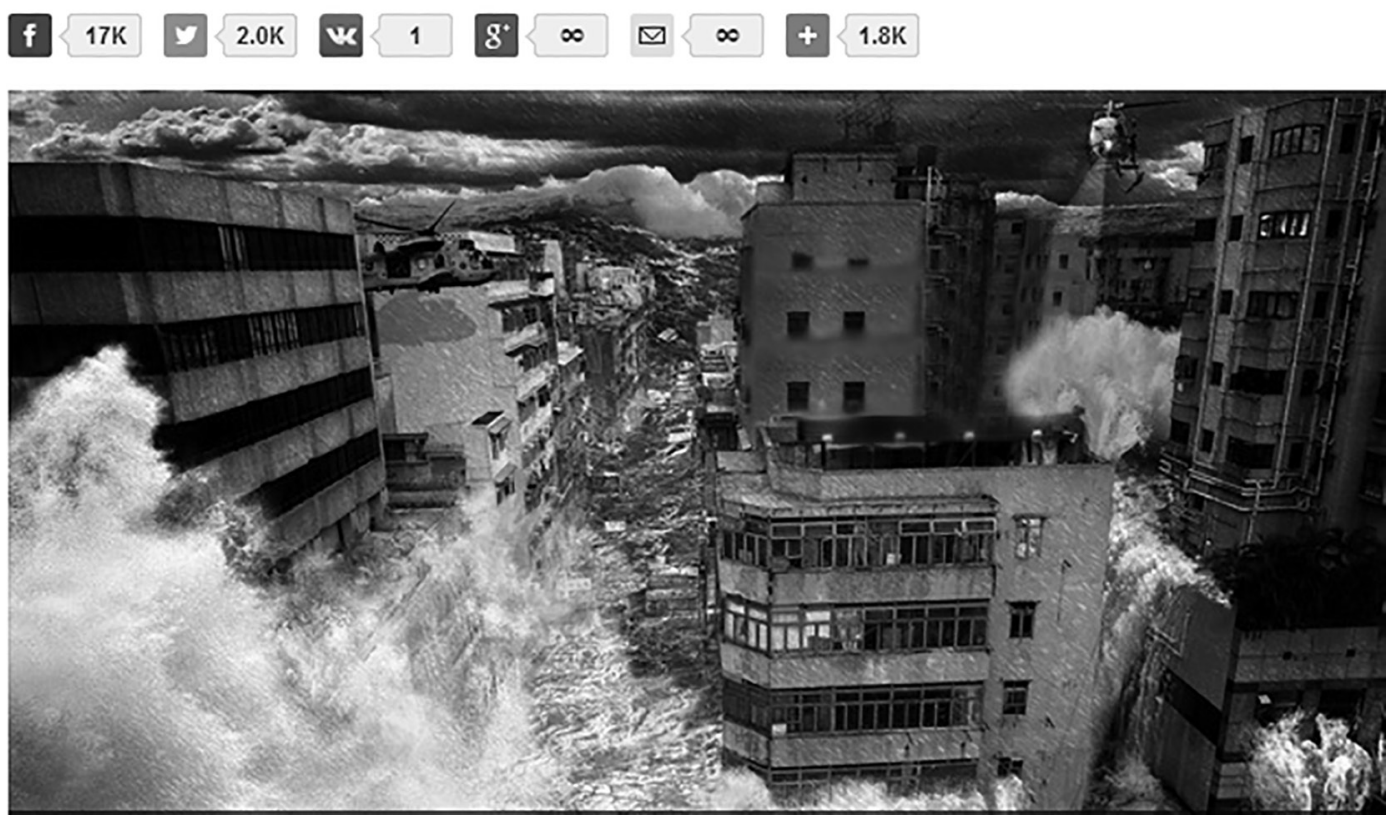

Fig. 1: Noticia, de corte sensacionalista-alarmista; sesga el resultado de investigaciones científicas que evaluaron un evento plausible. Exagera la circunstancia mediante una imagen manipulada, fuera de contexto, con el fin de llamar la atención y mejorar el “rating” del medio (RT-Actualidad, 2013).

un consenso ni una conclusión definitiva, más allá de toda duda razonable. Más adelante se ofrecerán otros detalles sobre este punto, en particular en lo que concierne el caso del terremoto de L'Aquila.

La observación, medición e interpretación de la acumulación de la deformación en la corteza terrestre, mediante el Global Positioning System -GPS- satelital y/o por medio de la geodesia de primer orden, correlacionada con la acumulación del estado de esfuerzos mecánicos y su posible liberación mediante procesos cinéticos y dinámicos, puede ser una señal precursora accesoria y complementaria valiosa.

Sin embargo, todavía no es suficiente, por sí sola, para discernir o anticipar con precisión y exactitud, cuál sería la falla tectónica que produciría el sismo, ni el tiempo, lugar, profundidad y magnitud (i.e. cantidad de energía sismogénica a liberar y su momentum) con que se materializaría el evento futuro posible. Las correlaciones empíricas, sobre las 
que se basa esta metodología, son todavía apenas referenciales y aproximadas y, a lo sumo, permiten conocer gruesamente el orden de magnitud de los parámetros sismogénicos en sus escalas espaciales y temporales.

Existen otras metodologías, sobre las cuales hay una extensa bibliografía, que aparecen como "comple-mentarias" y denominadas "precursores", como por ejemplo la observación del comportamiento de algunos animales, cambios en la relación de las celeridades de las ondas $\mathrm{P}$ y S, variaciones en el campo electromagnético, cambios en las propiedades elásticas y de la "dilatancia" (i.e. aumento del volumen, aún y cuando la roca se encuentra en condiciones de compresión) de los macizos rocosos.

También se incluyen, la ocurrencia de tipos especiales de sismos premonitores, caracterizados por su "signatura" anómala, la "brecha" o silencio sísmico previo, etc. Sin embargo, aparte de lo que parecen ser ca-sualidades o eventos fuera de las tendencias típicas ("outliers"), no parece haber una consistencia exitosa y verificable que permita presumir la validez de las hipótesis sobre las que se fundamentan las teorías respectivas que respaldan esas metodologías.

\section{ANTECEDENTES}

\section{L'Aquila}

L'Aquila fue fundada en 1254 y se encuentra a una elevación de $714 \mathrm{~m}$ sobre el nivel del mar. Es la capital de Abruzzo y de la provincia de L'Aquila; se encuentra en el centro de Italia, al noreste de Roma (Fig. 2). Tiene una población de alrededor de 73.000 habitantes y, por su actividad económica, alberga muchas industrias electrónicas.

La ciudad está rodeada de muros medievales y posee lugares de interés histórico y arquitectónico, como por ejemplo La Rocca Calascio, la fortaleza más alta de Italia y una de las más altas de Europa. También, el fuerte español del siglo XVI, construido bajo la dirección del ingeniero valenciano Pedro Luis Escrivá; la basílica románica de Sta. María de Collemaggio, la basílica de S.Bernardino y la Fontana medieval de los 99 chorros, que conmemora los 99 pueblos que, se dice, establecieron la ciudad. La Fontana Luminosa es otro lugar de interés: una escultura de dos mujeres que sostienen varios jarrones, hecha durante los años 1930.

En los alrededores de la ciudad hay ruinas romanas (Amiternum), monasterios antiguos y numerosos castillos. También hay pistas de esquí en el Gran Sasso d'Italia, la montaña más grande de los Apeninos.

\section{La sismicidad y la amenaza sísmica en Abruzzo}

En la región de Abruzzo, la sismogénesis se asocia a un sistema de fallas tectónicas activas en los montes Apeninos y posee un historial de sismos destructivos recurrentes. El evento de 1703, el más destructivo del que se tiene registros, tuvo un saldo de alrededor de 6.000 muertos.

Esta región está incluida dentro de una zona de peligro máximo, de acuerdo con la norma sísmica italiana (Figs 3 y 4) (Instituto Nazionale di Geofisica e Vulcanologia, 2003; Protezione Civile-Classificazione Sismica, 2003).

\section{Final de 2008 e inicio de 2009}

A partir de octubre de 2008 y hasta el principio de abril de 2009 , se produjo un enjambre sísmico en la región de Abruzzo. Dada la persistencia y frecuencia de los sismos durante este enjambre, la población de L'Aquila comenzó a alarmarse. En el cuadro 1 aparecen resumidos algunos factores relacionados con la percep-ción por parte de los actores e involucrados, de las incidencias, situaciones y resultados del proceso y evolución de los episodios previos y posteriores al terremoto del 6 de abril de 2009 en L'Aquila, Italia.

Posteriormente apareció, en los medios de comunicación y en el Internet, el Sr. Gioacchino Giampaolo Giuliani, técnico de los Laboratori Nazionali de Gran Sasso y aficionado a la sismología, quien no parece poseer calificaciones académicas específicas, según fuese calificado por el Istituto Nazionale di Astrofisica (2009). El 


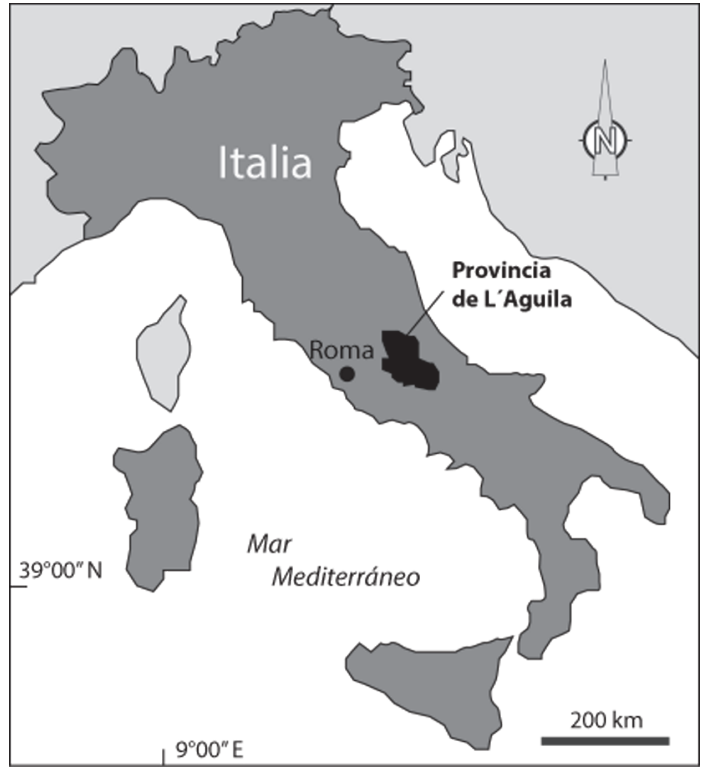

Fig. 2: Mapa de ubicación. L’Aquila, Abruzzo, Italia.

Sr. Giuliani y sus colegas (Giuliani et al., 2009; Pulinets et al., 2009) realizaron mediciones de las emisiones del gas radón provenientes del terreno y difundieron, por Internet y a la prensa, un índice de emanaciones y su correlación con un posible sismo próximo (Moreno, 2009).

Este método, controversial, no ha logrado demostrar su eficacia para predecir terremotos, pues en numerosos casos en el mundo se han detectado variaciones del radón y no se ha producido un sismo y, en otros casos también muy numerosos, han sucedido terremotos sin que se hayan registrado variaciones precursoras "anormales" del gas (Immè $\&$ Morelli, 2012). Esto explica que el Sr. Giuliani y otros como él, que han realizado estudios e intentos de predicción semejantes, hayan desacertado repetidamente en sus propósitos: “...the International Commission on Earthquake Forecasting for Civil Protection, after interviewing Giuliani, found that there had been no valid prediction of the main shock before its occurrence...". [“...la Comisión Internacional para el Pronóstico de Terremotos para la Protección Civil, luego de entrevistar al Sr. Giuliani concluyó que no hubo una predicción válida del evento principal antes de que sucediera...". Traducción del autor] (International Commission on Earthquake Forecasting for Civil Protection, 2011). La prensa ha tomado posiciones contradictorias e inconsistentes en cuanto a la cobertura
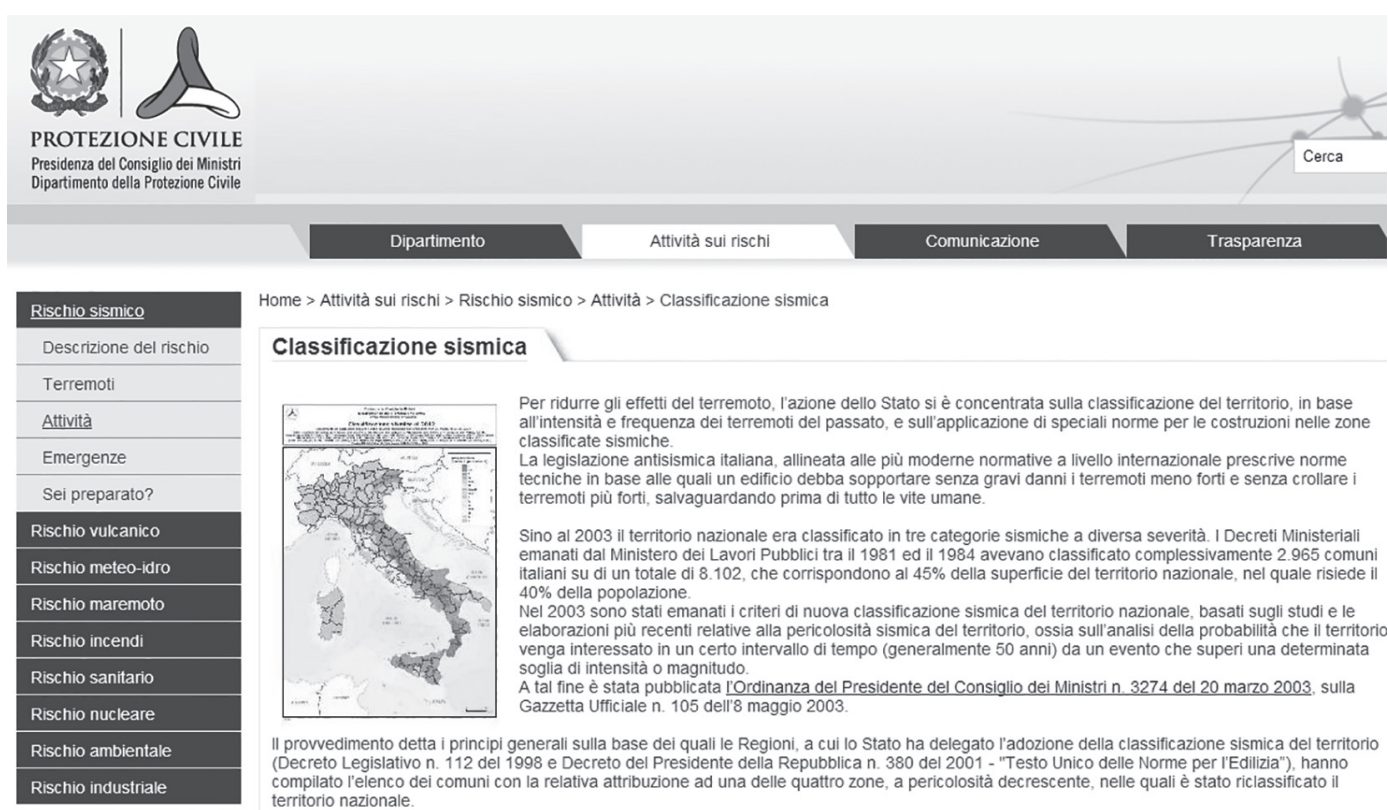

Home > Attività sui rischi > Rischio sismico $>$ Attività $>$ Classificazione sismica

Classificazione sismica

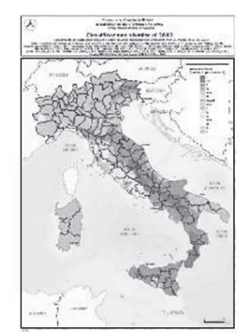
Per ridurre gli effetti del terremoto, l'azione dello Stato si è concentrata sulla classificazione del territorio, in base
all'intensità e frequenza dei terremoti del passato, e sull'applicazione di speciali norme per le costruzioni nelle zone classificate sismiche. La legislazione antisismica italiana, allineata alle più moderne normative a livello internazionale prescrive norme tecniche in base alle quali un edificio debba sopportare senza gravi danni i terremoti meno forti e senza crollare $i$ terremoti più forti, salvaguardando prima di tutto le vite umane.

Sino al 2003 il territorio nazionale era classificato in tre categorie sismiche a diversa severità I Decreti Ministeriali emanati dal Ministero dei Lavori Pubblici tra il 1981 ed il 1984 avevano classificato complessivamente 2.965 comuni italiani su di un totale di 8.102 , che corrispondono al $45 \%$ della superficie del territorio nazionale, nel quale risiede il $40 \%$ della popolazione.

Nel 2003 sono stati emanati i criteri di nuova classificazione sismica del territorio nazionale, basati sugli studi e le elaborazioni più recenti relative alla pericolosità sismica del territorio, ossia sull'analisi della probabilità che il territoric venga interessato in un certo intervallo di tempo (generalmente 50 anni) da un evento che superi una determinata

A tal fine è stata pubblicata l'Ordinanza del Presidente del Consiglio dei Ministri n. 3274 del 20 marzo 2003, sulla

II provvedimento detta i principi generali sulla base dei quali le Regioni, a cui lo Stato ha delegato l'adozione della classificazione sismica del territorio (Decreto Legislativo n. 112 del 1998 e Decreto del Presidente della Repubblica n. 380 del 2001 - "Testo Unico delle Norme per l'Edilizia"), hanno territorio nazionale.

Fig. 3: Imagen de acceso a la información sobre la Clasificación de la Amenaza Sísmica de Italia Mapa (Instituto Nazionale di Geofisica e Vulcanologia, 2003; Protezione Civile-Classificazione Sismica, 2003). 


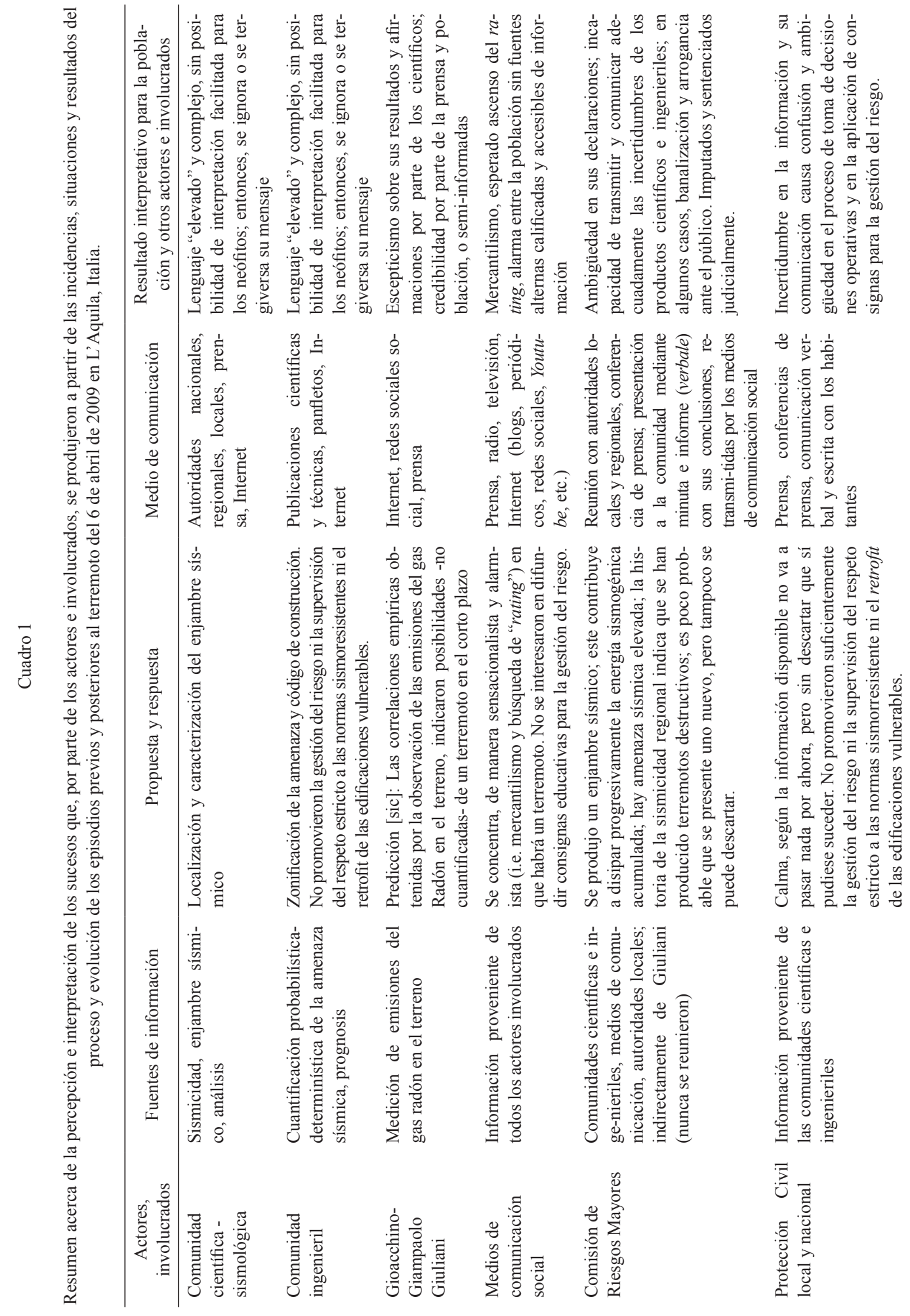




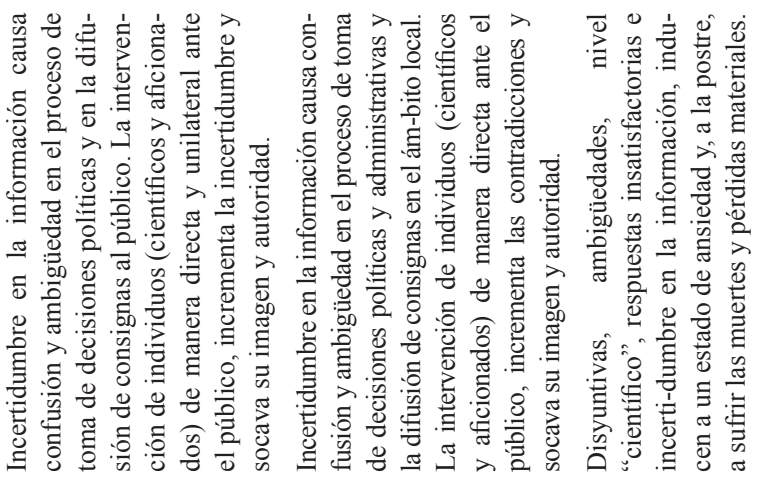

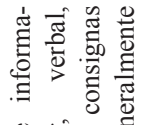

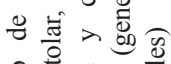

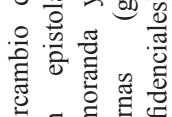

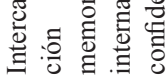

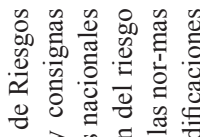

入 $\lambda$ 敌

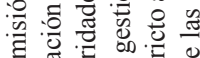

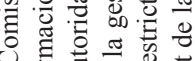

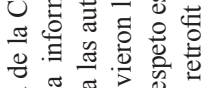

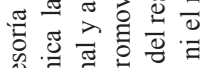

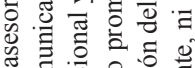

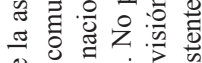

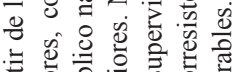

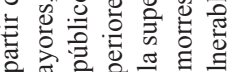

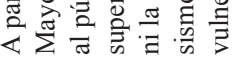

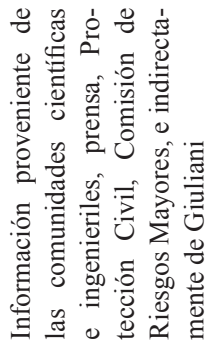

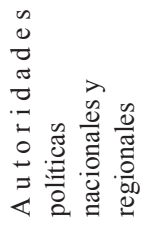

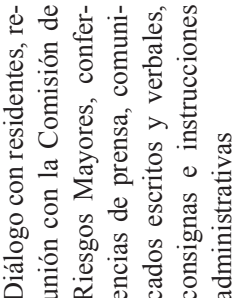

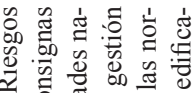

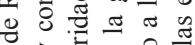

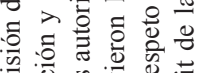

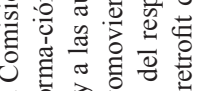

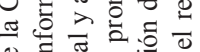

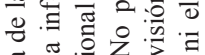

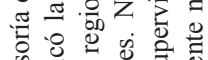

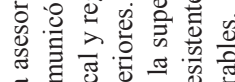

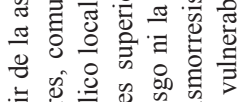

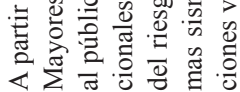

品官

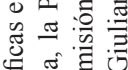

舒通

릉 흐

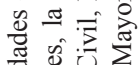

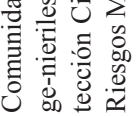

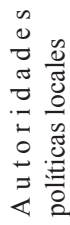

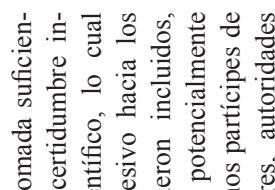

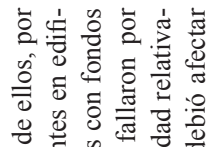

\%

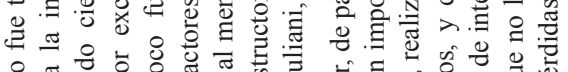

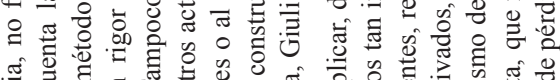

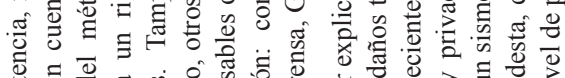

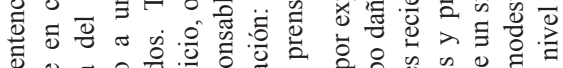

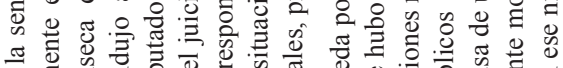

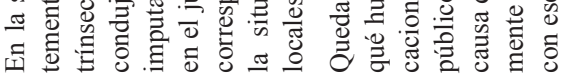

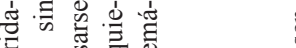

$\exists$ \&े

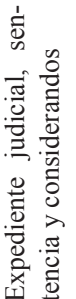

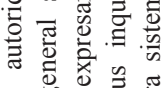

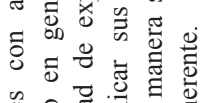

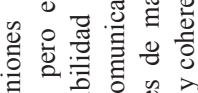

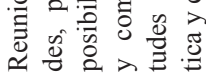

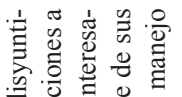

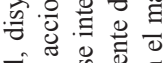

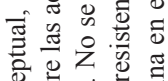

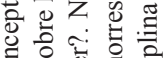

ôd

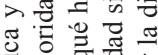

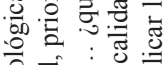

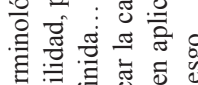

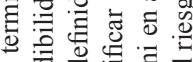

:

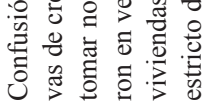

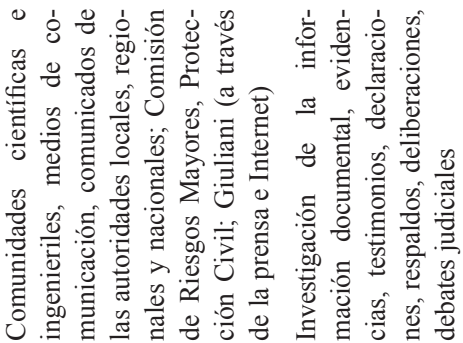

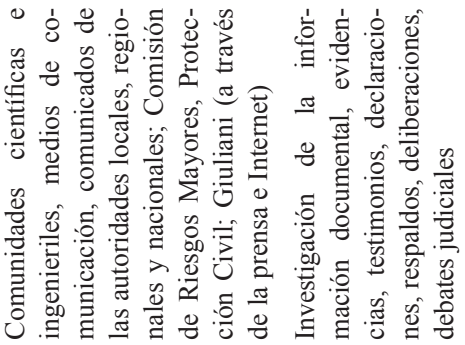

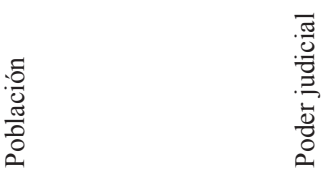

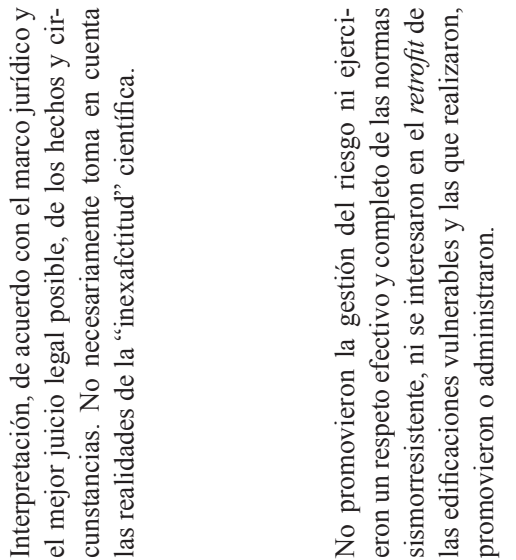
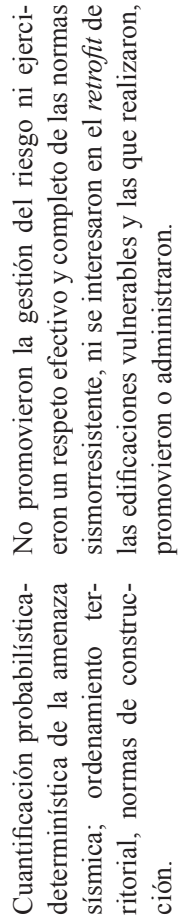

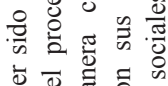

范。䒕

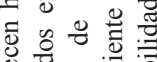

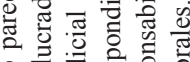

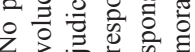




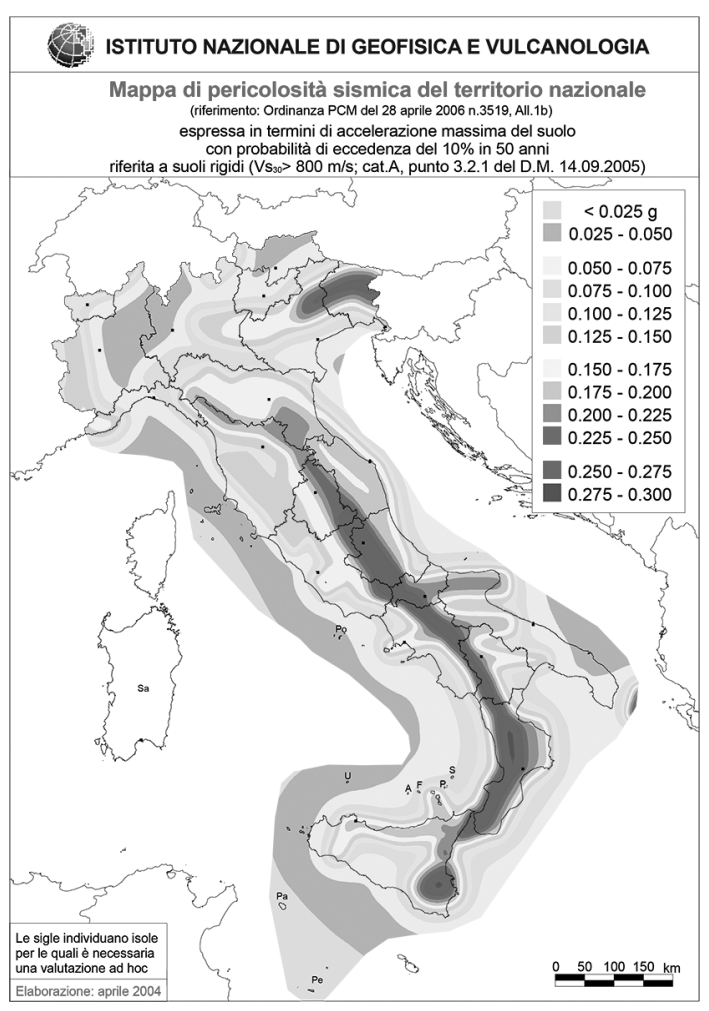

Fig. 4: Mapa de amenaza sísmica, Italia, expresada con la aceleración pico del terreno, una probabilidad de excedencia del $10 \%$ en 50 años y en un suelo rígido. La región de L'Aquila está indicada dentro de una zona con aceleración entre 0,25 y $0,275 \mathrm{~g}$ (Instituto Nazionale di Geofisica e Vulcanologia, 2003).

mediática que recibió el caso de la "predicción" del Sr. Giuliani, basada en sus observaciones del Radón (Fox, 2009), lo cual ciertamente contribuyó a la generación de confusiones en la opinión pública y en los tomadores de decisiones.

Curiosamente y en este caso, aunque hubiese podido haber "acertado" por casualidad su predicción (Greenemeier, 2013), parece que el detalle de las numerosas imprecisiones y fallos previos escapó o no importó a la prensa, cuyo énfasis se concentró en subrayar el contenido sensacionalista de su actuación. El resultado final fue asignarle un papel inmerecido de protagonista al Sr. Giuliani durante el imbroglio mediático que se produjo a alrededor del terremoto y del procedimiento judicial posterior. Con su sensacionalismo mercantilista, la prensa alimentó el estado de ansiedad y desinformación del público, lo cual, desafortunadamente ha sucedido con frecuencia en otros países, como por ejemplo en Centroamérica.

\section{Marzo de 2009, el comunicado público}

El 30 de marzo de 2009 sucedió un sismo de $\mathrm{M}_{\mathrm{w}} 4,0$ a las 11 p.m., lo cual motivó al Alcalde a ordenar la evacuación de varios edificios públicos y cerrar una escuela. Ante ello y aparte de algunas explicaciones inconcluyentes ofrecidas por la Asesora Regional de la Protección Civil el 31 de marzo de 2009 (Stati, 2009), posiblemente resultado de otras comunicaciones, a veces contradictorias, entre sus superiores (Caporale \& Dusi, 2012), el nerviosismo comenzó a imperar entre la población.

Esta situación fue causada por la insuficiencia y confusión entre las opciones y contradicciones relaciona-das con la información y respuestas a las cuales tenía acceso el público, tanto de parte de las autoridades nacionales (i.e. de orden político y administrativo), de la protección civil (i.e. operativas) y científicas (i.e. especialistas en sismología y amenaza sísmica). Progresivamente, fue generándose una situación difícil e incierta, igualmente para las mismas autoridades locales (Pritchard, 2012). Debido a esto, el gobierno italiano convocó a la Comisión de Riesgos Mayores a una reunión el 31 de marzo, en L'Aquila. Estuvieron presentes autoridades del gobierno local, funcionarios de la Protección Civil y seis expertos en geociencias e ingeniería. No hay información acerca de si fue discutido el caso, ni del porqué no estuvo presente el Sr. Giuliani, quien no fue interrogado acerca de su medición de las 


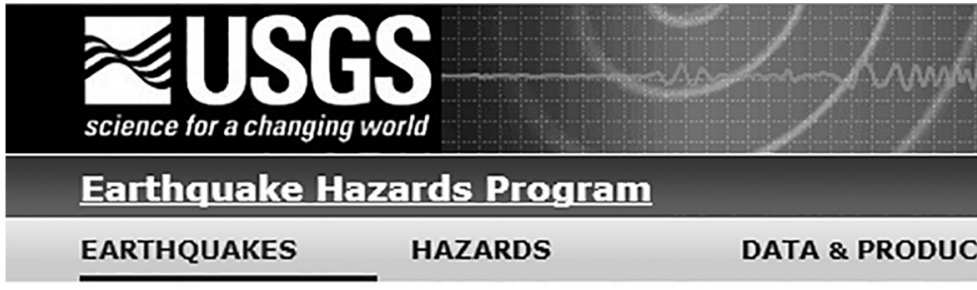

ShakeMap

\section{Shakemap us2009fcaf}

USGS ShakeMap : CENTRAL ITALY

Mon Apr 6, 2009 01:32:39 GMT M 6.3 N42.33 E13.33 Depth: 8.8km ID:2009fcaf

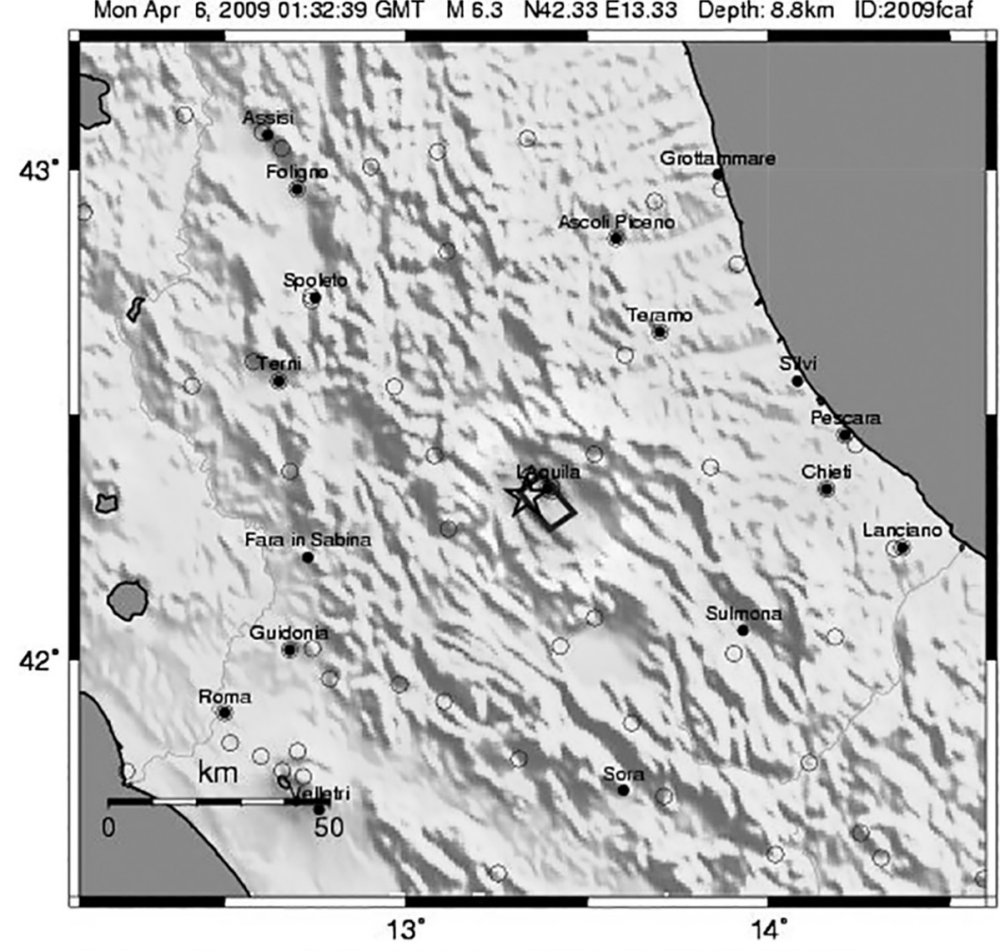

Map Version 7 Proceseed Wed May 20, 200909:43:43 AM MOT - NOT REVIEWED BY HUMAN

\begin{tabular}{|c|c|c|c|c|c|c|c|c|c|}
\hline $\begin{array}{l}\text { PEACENEED } \\
\text { SHAKIMG }\end{array}$ & Not felt & Weak & Light & Moderate & Strong & Very strong & Severe & Violent & Extreme \\
\hline $\begin{array}{l}\text { POIEMIAL } \\
\text { DAMAGE }\end{array}$ & none & none & none & Very light & Light & Moderate & ILoderatisheavy & Heavy & Very Heavy \\
\hline PEAK ACC (\%) & $<.17$ & $.17-1.4$ & $1.4-3.9$ & $3.9-9.2$ & $92-18$ & $18-34$ & $34-65$ & 65-124 & $>124$ \\
\hline PEAK VEL(cmb) & $<0.1$ & $0.1 \cdot 1.1$ & 1.1-3.4 & $3.4-8.1$ & $8.1-18$ & $18 \cdot 31$ & 31.60 & 60.116 & $>118$ \\
\hline $\begin{array}{l}\text { IRSTRUMERTAL } \\
\text { IMTEMSTYY }\end{array}$ & I & IHIII & IV & V & VI & VII & VIII & $\bar{x}$ & $x_{t}$ \\
\hline
\end{tabular}

Fig. 5: Epicentro e isosistas del terremoto del 6 de abril de 2009, 01:32:39 UTC, $M_{w}$ 6,3. IMMmáx. VIII-IX; aceleración máxima estimada en 60\%. (USGS-EHP; 2013). 
emisiones del gas radón, su índice de emanaciones, su correlación y afirmaciones sobre la proximidad de un posible sismo.

Los temas abordados en la reunión, de una hora, fueron documentados en una minuta de dos páginas (Commissione Grandi Rischi, 2009; Caporale \& Dusi, 2012) y en un "verbatim" (verbale) de cuatro páginas (Departamento della Protezione Civile, 2009; L'Espresso, 2009).

Después de la reunión, el vocero Bernardo De Bernardinis, Subjefe Operativo Nacional de la Protección Civil, informó al público las conclusiones: “...no hay razón para pensar que la secuencia de sismos, de baja magnitud, como la que se presenta, sea premonitora de un evento mayor..." (Ortolani \& Spizuoco, 2010). Tam-bién indicó que no es posible predecir los sismos y que el enjambre había generado aceleraciones muy bajas, las cuales solo provocan daño a las estructuras frágiles. Por otra parte afirmó que “...la comunidad científica considera favorable el proceso continuo de liberación de energía y, por lo tanto, de haber un evento futuro, este no será demasiado intenso...".

Un periodista le preguntó, de seguido, si la gente podía regresar a dormir a su casa y tomar una copa de vino; él contestó que no había peligro inminente y sugirió incluso una marca de vino de la región (Hall, 2011; Pritchard, 2012).

Luego de esto, muchas familias decidieron suspender la precaución de dormir fuera del centro histórico, en casas móviles, carpas o albergues, como lo habían hecho hasta entonces.

\section{EI terremoto}

Siete días más tarde, el 6 de abril de 2009 sucedió el terremoto de L'Aquila a las 00:32 horas, con una magnitud de momento $\mathrm{M}_{\mathrm{W}}$ 6,3 (United States Geological Survey-Earthquake Hazard Program, 2009; Fig. 5), con una profundidad de $8,8 \mathrm{~km}$ y producido por una falla normal $\mathrm{N}_{\mathrm{W}}$-SE. Su epicentro se encontró a $8 \mathrm{~km}$ al SW de L'Aquila, tuvo una intensidad máxima IMMmáx $=$ VIII-IX y una aceleración máxima estimada $\mathrm{PGA} \approx 60 \% \mathrm{~g}$.
El saldo del evento fue de 309 muertos, 1.500 heridos, la destrucción de numerosos edificios en el centro histórico y también de algunas residencias estudiantiles y de un hospital, estos últimos construidos recientemente. Alrededor de 67.000 personas perdieron sus viviendas (Figuras 6, 7 y 8 ). En la literatura consultada no fue posible encontrar una estimación de los daños y pérdidas económicas y financieras causadas por el terremoto.

\section{EL JUICIO Y SUS SECUELAS}

\section{Acusación}

Luego del terremoto del 6 de abril, los expertos y el subjefe de Protección Civil fueron acusados ante los tribunales de justicia. La Fiscalía Penal (Tribunale de l'Aquila, 2012) les imputó, entre otras cosas, haber ofrecido información incompleta, imprecisa y contradictoria y realizado una valoración aproximada, genérica e ineficaz del riesgo, lo cual interfirió con la ejecución de medidas de previsión y prevención, brindado información incompleta, imprecisa y ambigua a las autoridades y a la ciudadanía sobre la naturaleza, causa, peligrosidad y evolución de la sismicidad regional: “...alla cittadinanza aquilana, informazioni incomplete, imprecise e contraddittorie sulla natura, sulle cause, sulla pericolosità e suifuturi sviluppi dell'attività sismica in esame, in tal modo vanifcando le finalità di "tutela dell'integrità della vita, dei beni, degli insediamenti e dell'ambiente dai dannio dal pericolo di danni derivanti da calamità naturali, da catastrofi e da altri grandi eventi che determinino situazioni di grave rischio...".

También, les imputó haber alegado que la sismicidad no se puede predecir, que es difícil estimar su evolución y que la simple observación de un enjambre no permitía concluir si este iba o no a ser precursor de un terremoto [...] haber dicho que los terremotos en Abruzzo tienen períodos de recurrencia muy largos y que era improbable que se presentara uno similar al de 1703, aunque esto no se podía asegurar de manera absoluta: “... 


\section{The L'Aquila earthquake}

Early Monday morning, central Italy was struck by a 6.3 magnitude earthquake. The 2009 L'Aquila earthquake caused serious damage to several medieval hill towns in the region, killing over 260 residents, injuring over 1,000 and leaving 28,000 homeless. Despite the dangers from aftershocks, the search for survivors continues, and will be maintained until Sunday, after which the daunting tasks of cleanup and reconstruction will begin. (32 photos total)

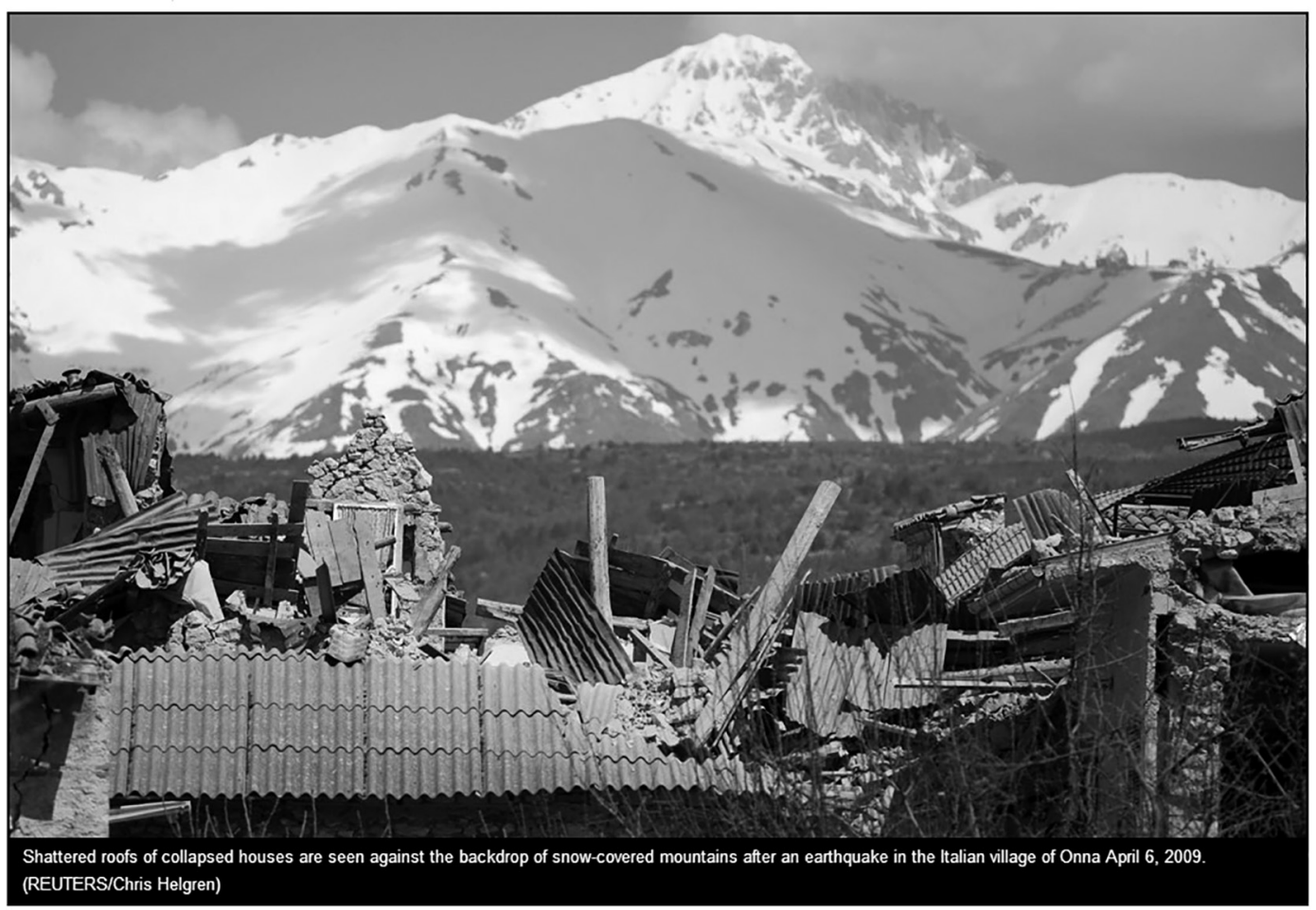

Fig. 6: L'Aquila, Italia. Aspecto de los daños causados por el terremoto del 6 de abril de 2010, $M_{w}$ 6,3. En el plano posterior, los montes del Gran Sasso (The Big Picture, 2009).

affermando che sui terremoti "non è possibile fare previsioni", "è extremamente difficile fare previsioni temporali sull'evoluzione dei fenomeni sismici", "la semplice osservazione di molti piccoli terremoti non costituisce fenomeno precursore" $e$ al contempo l'esatto contrario ovvero "qualunque previsione non ha fondamento scientifico"; ritenendo che "i forti terremoti in Abruzzo hanno periodi di ritorno molto lunghi. Improbabile il rischio a breve di una forte scossa come quella del 1703, pur se non si può escludere in maniera assoluta"; ritenendo che "non c'è nessun motivo per cui si possa dire che una sequenza di scosse di bassa magnitudo possa essere considerata precursore di un forte evento...".
Adicionalmente y según el Fiscal, todo esto indujo a la comunidad a permanecer en sus casas, a pesar de que los sismos se presentaban con frecuencia y magnitud crecientes.

\section{Sentencia y condena}

Por los hechos imputados, los acusados fueron declarados culpables de lesión y homicidio culposo, condenados a seis años de cárcel, a resarcir sumas millonarias por las pérdidas económicas y patrimoniales y a ser inhabilitados para ocupar cargos públicos (Tribunale de l'Aquila, 2012; La Reppublica, 2013). Según el juez Marco 
theguardian TheObserver

News $\mid$ Sport $\mid$ Comment $\mid$ Culture $\mid$ Business $\mid$ Money $\mid$ Life \& style News > World news > Italy

Series: Dispatch

L'Aquila's earthquake-scarred streets see battle between science and politics

Convictions of six seismologists over 308 deaths three years ago have shocked colleagues - but not the disaster's survivors

Tom Kington in L'Aquila

The Observer, Saturday 27 October 201219.19 BST

国 Jump to comments (93)

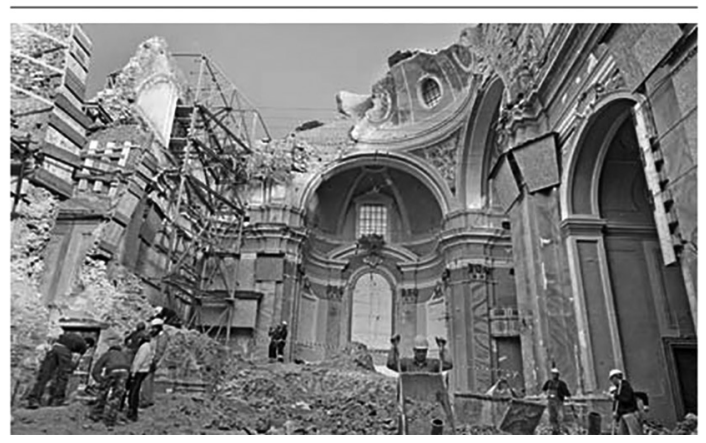

Fig. 7: El centro histórico de L'Aquila, afectado por el sismo del 9 de abril de 2009 (Kington, 2012).

Billi, no se demostró, sin embargo, que los acusados hubiesen hecho manifestaciones contrarias a su mejor criterio o que hubieran estado inspirados por intereses personales. El juez declaró que el fondo del asunto en la sentencia no era si habían fallado en predecir o anticipar el sismo, sino en su negligencia al investigar la situación de manera más concreta y por brindar información que indujo hacia una interpretación incorrecta e insuficiente, lo cual influyó posteriormente en la toma de decisiones erróneas. Sin embargo, la prensa informó, enfáticamente, que el fundamento de la condena se basó en que "los expertos no predijeron el sismo", por lo que muchos científicos, en el ámbito mundial, se manifestaron airadamente contra el fallo (Figs 8, 9 y 10). Como dato colateral, el técnico en gas Radón, Sr. Giuliani, aunque mencionado en el juicio y en la información mediática, no fue imputado, ni llamado como testigo.

\section{ANÁLISIS, DISCUSIÓN Y REPERCUSIONES}

\section{Responsabilidad colectiva e individual}

La ciencia sismológica es inexacta y sobre todo, lo son sus instrumentos predictivos. Su labor y productos se basan en el análisis de datos mediante herramientas estadísticas, cálculo y retro-cálculos de probabilidades, correlaciones retrospectivas, extrapolaciones, proyecciones, la aplicación de modelos, escenarios estocásticos y análisis empíricos. La predicción sísmica de corto plazo (horas, días, meses, años), es un reto que está muy lejos de resolverse, por lo que es imprudente dar crédito a este tipo de ejercicios.

Sin embargo, es importante señalar que el verbatim-il manifesto (Departamento della Protezione Civile, 2009), que complementa la minuta de la reunión del 31 de marzo (Comissionne Grandi Rischi, 2009), mostró que en realidad sí habían sido abordado algunos conceptos y criterios más precisos y relacionados con la ame-naza sísmica, de manera más realista que lo que el vocero comunicó a la prensa. Por ejemplo, uno de los expertos enfatizó que la actividad sísmica en L'Aquila resultaba de su ubicación entre dos placas tectónicas, que no podía descartarse un nuevo evento como el de 1703 y que un estudio realizado en 1995 había determinado la posibilidad de que se produjera un sismo de, al menos, $\mathrm{M}_{\mathrm{w}}$ 5,9 durante los siguientes 20 años. La amenaza sísmica en Abruzzo había sido estudiada con detalle y la normativa sismorresistente vigente prescribía la máxima rigurosidad para el diseño de las estructuras y de las construcciones en esa región (Instituto Nazionale di Geofi-sica e Vulcanologia, 2003; Protezione Civile; 2003).

Aun así, las autoridades locales parecieran no haber vigilado, con suficiente celo, el cumplimiento del código de construcción durante el diseño y la realización de las edificaciones nuevas, lo cual causó que algunas de ellas fuesen destruidas. Tampoco promovieron el refuerzo y readecuación de las edificaciones históricas. Por su parte y, de acuerdo con la información 
The

Economist World politics Business \& finance Economics Science \& technology Cultı

L'Aquila's earthquake

\section{Scientists in the dock}

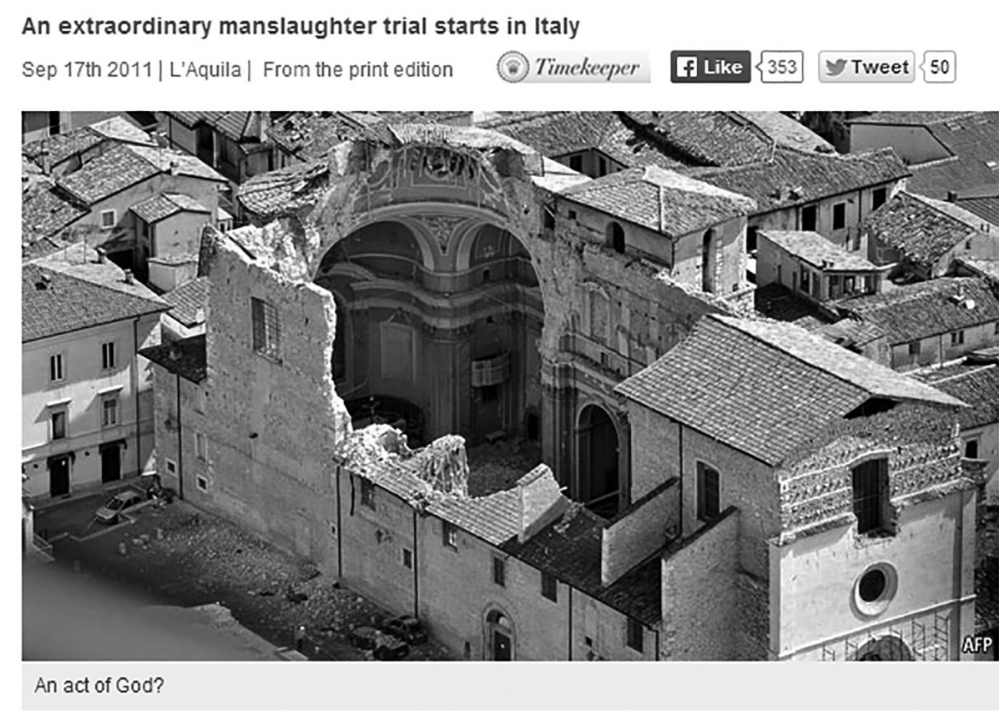

ON MARCH 31st 2009 Bernardo De Bernardinis, then deputy chief of Italy's Civil Protection Department, told people in and around the medieval Italian city of L'Aquila that a series of tremors which had been felt in the area over the past four months posed "no danger". Speaking to a journalist from a local television station, he said that "the scientific community continues to confirm to me that in fact it is a favourable situation, that is to say a continuous discharge of energy." Six days later L'Aquila and several surrounding villages lay in ruins. An earthquake of magnitude 6.3 had destroyed thousands of buildings and killed 308 people.

Fig. 8: Información periodística relacionada con la sentencia sobre el caso del juicio sobre el terremoto del 6 de abril de 2009 en L'Aquila, Italia (The Economist, 2011).

periodística consultada, la Protección Civil no se preocupó suficientemente por fortalecer la capacidad local para la gestión del riesgo, ni en la preparación para enfrentar contingencias de este tipo. Pero además, el vocero de la reunión del 31 de marzo, Sr. De Bernardinis, parece haber subestimado el peligro en su comunicado y no recordó a las personas su obligación de mantenerse preparadas, por lo menos por el hecho de vivir en una región ya catalogada, desde hace tiempo, como de elevada peligrosidad sísmica. Tampoco aludió la posibilidad de que el enjambre sísmico ya hubiese afectado o debilitado algunas edificaciones antiguas, con lo que redujo el interés e iniciativas por revisarlas y mejorar su desempeño.

Antes del enjambre y del terremoto, la prensa tampoco se había interesado en concienciar a la población acerca de las posibles deficiencias estructurales de las edificaciones y de sus servicios conexos de agua, gas, electricidad. Tampoco aprovechó la ocasión para manifestar la necesidad de tomar medidas de preparación y prevención, ni ejerció presión sobre las autoridades para prestarle atención a la vulnerabilidad de la comunidad. 


\section{lanacion $\cdot$ com \\ El Mundo \\ Últimas noticias \\ Secciones - \\ Edición impresa - \\ Blogs \\ LN Data \\ lanacion.com | El Mundo \\ Lunes 22 de octubre de 2012 | 17:21 \\ Seis años de prisión a científicos italianos por no predecir un terremoto}

Siete sismólogos fueron condenados por "homicidio involuntario" por no alertar el sismo de L'Aquila en 2009 que mató a 308 personas, pese a los temblores previos registrados

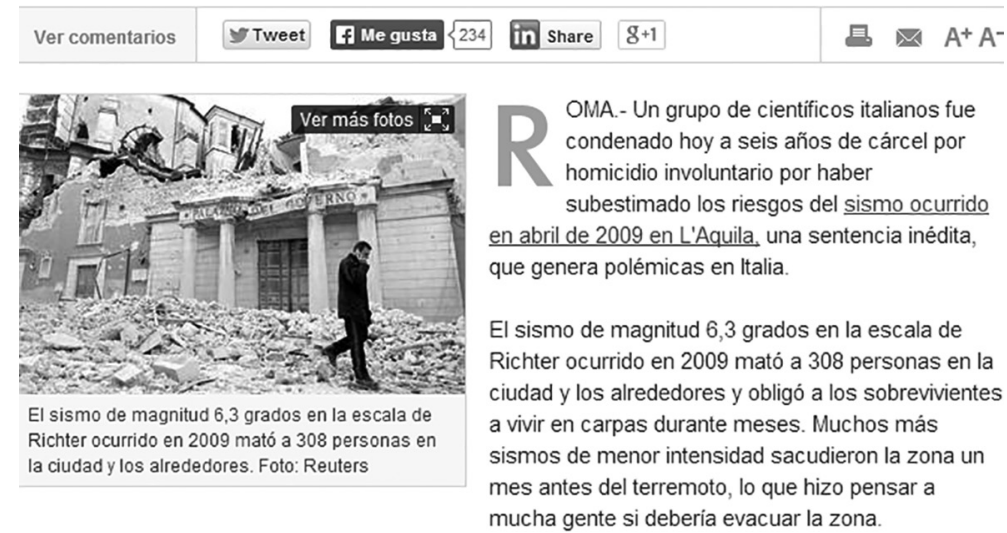

Fig. 9: Noticia acerca de la sentencia sobre el caso del juicio sobre el terremoto del 6 de abril de 2009 en L'Aquila, Italia. El énfasis se enfocó en que la sentencia se fundamenta en la "incapacidad de predecir sismos" (La Nación, 2012).

Por otra parte, el Sr. Giuliani y su medición del Radón acapararon la atención mediática, a pesar de haber "pronosticado" desacertadamente varios eventos anteriores. Pero como en el caso de L'Aquila "acertó", se convirtió de inmediato en un protagonista titular de la prensa por muchos días, la cual rápidamente olvidó y no puso en tela de duda sus fallos previos. La prensa parece no haberse tomado la molestia de estudiar a fondo los conside-randos y detalles expuestos durante el proceso judicial, tergiversó la sentencia y volcó a la opinión pública cali-ficada en contra del fallo.

Los expertos de la Comisión, por su parte, invocaron el "principio precautorio" y giraron una advertencia prudente, pero ambigua y que fue interpretada como un mensaje definitivo de calma. El mensaje oficial del 31 de marzo que llegó a la población, fue interpretado de manera, quizás excesiva, como de calma y confianza, lo cual la condujo hacia el efecto perverso de haberle hecho perder el interés por su seguridad individual y por la gestión del riesgo en general. Paradójicamente, sin tomar en cuenta las premoniciones del $\mathrm{Sr}$. Giuliani, insisten-temente resonadas por la prensa, los pobladores tampoco reaccionaron unilateralmente, no revisaron sus viviendas ni tomaron medidas para reforzarlas. Aparte de ello, bajaron la guardia y dejaron de aplicar las medidas preventivas que, aunque tímidas, habían estado tomando (e.g. dormir en casas móviles, carpas y albergues fuera del centro histórico, asegurarse que su ubicación no estuviera expuesta a peligros colaterales, etc.), lo cual debió continuar, al menos hasta que sus viviendas fuesen evaluadas $\mathrm{y}$, de 


\section{Gazzetta del Sudline}

Cerca
nel sito:

Fig. 10: En esta referencia, el periódico indica que la sentencia contra los científicos involucrados en el caso del terremoto del 6 de abril de 2009 en L'Aquila, Italia, se asemeja al caso de Galileo Galilei (Gazzeta du Sud, 2012).

ser el caso, reforzadas. A pesar de la exis-tencia de mapas de amenaza sísmica actualizados, guías para formular planes de respuesta y evacuación, y de la información disponible acerca del enjambre sísmico en sí mismo, estos recursos disponibles no fueron utilizados, aprovechados, ni aplicados.

Pareciera, según Hall (2011), que en algunos casos, algunos profesionales y empresas de construcción no habían aplicado las normas sismorresistentes en las edificaciones nuevas. Esto quedó demostrado, entre otras cosas, con el colapso de las residencias estudiantiles y del hospital, recientemente construidos con fondos públicos y cuyos daños fueron causados por deficiencias en el diseño estructural y por el uso de materiales y técnicas constructivas inadecuadas (La Reppublica, 2013). A pesar de que para la reconstrucción ya se han propuesto normas constructivas y un ordenamiento urbano más estricto, pareciera que estas todavía no se han llevado a la práctica.

\section{Aspectos políticos, legales y paradojas}

El código penal italiano, Art. 658 (Codice Penale, 2013) señala que: “...Procurato allarme presso l'autorità. Chiunque, annunziando disastri, infortuni o pericoli inesistenti, suscita allarme presso l'autorità o presso enti o persone che esercitano un pubblico servizio, è punito con l'arresto fino a sei mesi o con l'ammenda da euro 10 a euro 516...". (“...cualquiera que anuncie desastres, infortunios o peligros inexistentes y con cuya información alarme a la autoridad, a los entes y a las personas, o presione a quienes ejercen un servicio público, será penado con arresto de hasta seis meses o con una multa de 10 a 516 euros..." (Traducción del autor). Refiriéndose a este asunto, en febrero de 2013 y a partir de un twitter, mal interpretando informes del Istituto Nazional de Geofisica de que podría presentarse un terremoto, 


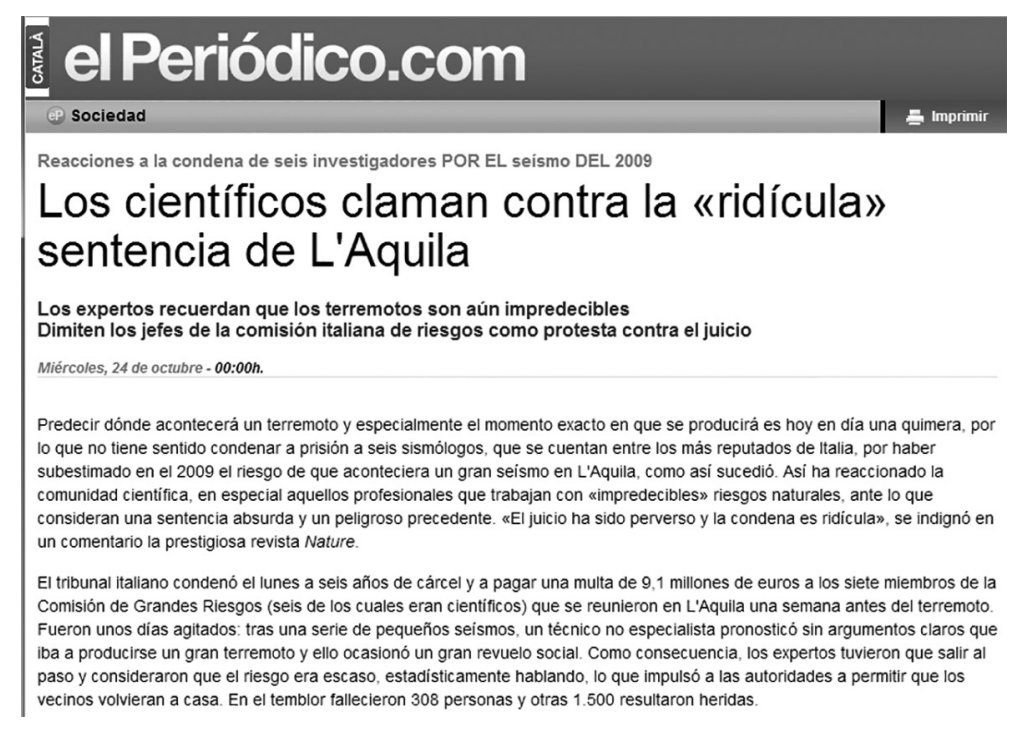

Fig. 11: Según El Periódico (2012), los científicos consideran "ridícula” la sentencia sobre el juicio del terremoto de L’Aquila.

el Director de la Protección Civil ordenó activar el estado de alerta y la evacuación de la población de Garfagnana, Toscana (Corriere della Sera, 2013). A causa de esto, cientos de personas abandonaron sus casas... y el sismo no sucedió, por lo que precisamente y con fundamento en ese artículo 658, el Director -hoy ex-Director- fue condenado por haber generado una falsa alarma. Entonces, “...si se alarma a la población se es condenado -como en Garfagnana- y si no se alarma, también...”, indicó uno de los defensores en el caso de L'Aquila (Grennemeier, 2013).

Ahora bien, si la condena prospera luego de la apelación (Nosengo, 2013), en adelante, la posición más conservadora ante una situación anómala, ¿será guardar silencio total? (Pritchard, 2012); o por el contrario, ¿desconocer las limitaciones de la ciencia, no ser honesto y decir que sí va haber un terremoto, aunque no lo haya? Esta sería la posición más cómoda y segura, pues por eso a nadie lo condenarán. Pero ¿qué hacen luego las autoridades de la protección civil?, ¿ignorar cualquier síntoma de un desastre?, ¿evacuar comunidades enteras sin mayor sustento?, ¿o no actuar del todo?

La consecuencia inmediata de esta ambivalencia será, posiblemente, la resistencia de los expertos serios a compartir sus avances de investigación y prognosis, advertencias y acción preventiva, lo cual no es prometedor para el esfuerzo de crear resiliencia. Pero tampoco se trata de que los "científicos" difundan a rienda suelta y, por sí solos, información crítica que debe más bien vincularse con la seguridad pública. Eso es tarea de las autoridades competentes, de sus asesores para la gestión del riesgo y de los especialistas en comunicación social. La ciencia se hace en los laboratorios $\mathrm{y}$ en el terreno, de manera multidisciplinaria; por su parte, la gestión del riesgo es la que se orienta hacia la política pública y la acción con las comunidades. Si no se hacen en equipo, de manera coordinada y complementaria, de nada le sirven a la sociedad, quien con sus impuestos las sustenta, sobre todo con el objetivo de recibir información y respuestas claras a sus interrogantes. Es imperativo recordar, constantemente, que la comunidad siempre está a la espera de indicaciones y consignas viables, comprensibles y accesibles, para satisfacer así sus necesidades de información y orientar adecuadamente sus propias decisiones sobre la seguridad, tranquilidad y su vida cotidiana.

El análisis del Comité de Riesgos Mayores de Italia (Commissione Grandi Rischi, 2009), de reconocida reputación científica, se realizó presumiblemente bajo presión e incertidumbre, pero 
el juez dictaminó que produjo “...información incompleta, imprecisa y contradictoria...". El juez no aceptó que un sismo intenso puede presentarse, o no, luego de una serie de posibles signos premonitores. Tampoco admitió que pudiese descartarse que sucediera, o que sencillamente la ciencia, con todos sus desarrollos actuales, aún no es capaz de pronosticar los terremotos con precisión. El Comité fue convocado para opinar ante una predicción "diletante" y su pecado fue señalar que no era posible confirmarla ni descartarla. La respuesta del Comité se obtuvo luego de una reunión de apenas una hora, quizás con poca modestia frente al protagonismo de un aficionado, cuyo canal para expresar su "certeza" no fue debatir con el Comité, sino haber utilizado los medios de prensa, quienes por supuesto acogieron sus vaticinios -quizás bien intencionados- de manera sensacionalista, alarmista y sobretodo mercantilista. En realidad, si el sismo sucedía o no, era irrelevante, pues lo realmente importante hubiera sido asegurarse que la calidad de las construcciones era la adecuada para enfrentar la amenaza símica prevalente, ilustrada claramente en los mapas existentes, en los estudios realizados y en las normas sismorresistentes. De nuevo, la presión de la prensa, sobre todo después del juicio, contribuyó a exacerbar los ánimos y a atizar la hoguera en contra de los científicos (The Economist, 2011), quienes de todas maneras y lógicamente han proce-dido a apelar la sentencia (Euronews, 2012)

\section{ENSEÑANZAS PARA AMÉRICA CENTRAL}

En opinión del autor, la sentencia no parece justa y es más bien un precedente grave, pues contribuye a alimentar el síndrome de buscar culpables. De este evento podría desprenderse una lección importante y es que, en la gestión del riesgo, debe prevalecer el criterio de la responsabilidad compartida, tanto por lo que se hace, como por lo que se deja de hacer por parte de todos los involucrados: Comités de alto nivel, autoridades locales, prensa, comunicadores, científicos, ingenieros, técnicos, población, constructores, urbanistas y jueces. Peor aún, puede también desembocar en la necesidad de invertir, a toda costa y sin miramientos, recursos infinitos en la predicción de eventos que no pueden ser predichos (Greenemeier, 2011), mientras que se dejan de lado y se eclipsan otras prioridades mayores, como por ejemplo que los edificios nuevos no fallen con un sismo modesto. Aunque un sismo de $M_{w} 6,3$ puede producir intensidades elevadas, no debería generar daños severos si se res-petan las normas sismorresistentes básicas.

La comunidad sismológica e ingenieril nunca podrá negar la posibilidad de que suceda un nuevo terremoto en cualquier parte de los territorios expuestos a la amenaza sísmica. Por lo tanto, es más importante enfocar los recursos de los países hacia la educación, diseño y construcción de buena calidad y a la gestión del riesgo que en las predicciones y pronósticos, pues ya sabemos que de todas maneras los sismos seguirán sucediendo; cómo los afrontemos con nuestra gestión del riesgo será lo que marque la diferencia.

Es claro, entonces, que la noción de la "baja probabilidad" de un evento destructivo no puede confundirse con que es "improbable" que suceda. De la misma manera, el período de recurrencia elevado de un evento no significa que es poco probable que suceda pronto y antes de su "vencimiento". Además, el hecho de que "la ley" no acepte los conceptos de probabilidad, grado de verosimilitud, los tipos y fuentes de la incertidumbre y que la lógica sea aún aristotélica o binaria, no debe generar prejuicios entre los jueces ni amedrentar la posición del científico.

Tampoco se trata de respaldar a unos amigos frente al juez, sino aclarar el peso real del conocimiento, la falibilidad e imprecisiones intrínsecas del método científico y del esfuerzo que se debe continuar para avanzar en procura de proteger a la sociedad. La nota de descargo publicada por el Dr. Enzo Boschi (Boschi, 2013) muestra claramente estas contradicciones y sobre todo, la comunicación disfuncional entre la Justicia y la ciencia, con los científicos atrapados en el medio de una polémica que parece estancada en un nudo gordiano: “...In publishing an official map, seismologists have done all they currently can to protect society from earthquakes. I can hardly be blamed for the poor quality of buildings or for people's failure to conform to anti-seismic laws- 
these are the responsibilities of other authorities. The local CPA is responsible for accurate communication of risk and effective management of emergency situations. I did not disseminate false or imprudent information. My question is: What could I do to avoid conviction? I suppose I should have foreseen the earthquake!..." ("...al publicar un mapa oficial, los sismólogos hicieron todo lo que han podido para proteger, de los terremotos, a la sociedad. No puedo ser culpado por la calidad pobre de las edificaciones o de la incapacidad de la gente al cumplir o no con las normas antisísmicas - esta es la responsabilidad de otras autoridades-. La autoridad local de la Protección Civil es la que debe responsabilizarse de una comunicación precisa de la gestión efectiva del riesgo y de las situaciones de emergencia. Yo no diseminé información falsa ni imprudente. Mi pregunta es entonces: ¿Qué debí haber hecho para evitar ser sentenciado? Supongo que haber anticipado el sismo (Boschi, 2013. Traducido por el autor).

Pero entonces, ¿no es paradójico que si alguien predice un gran terremoto y no acierta, no lo metan a la cárcel? Al contrario: todos agradecen su desacierto. Eso sí, quedará libre para seguir con sus cábalas, hasta que un día el azar y la coincidencia lo dejen bien parado y reconocido como adivino por su "acierto", seguramente gozando de su fama y de su ego, aunque la evidencia de su éxito se convierta luego en un gran desastre para la comunidad (Rojas, 2013).

Además, en el caso de L’Aquila y según la opinión manifestada por penalistas, científicos, ingenieros italianos e internacionales y la prensa, parece ser que no todos los posibles culpables fueron llevados a juicio. Faltaron, entre otros, algunas autoridades públicas nacionales y locales, empresas constructoras, corredores de bie-nes raíces y algunos medios de comunicación (The Economist, 2011).

Entonces, no hay que ver este caso como lejano para nuestros países, pues implica una gran responsabilidad por parte de las comunidades científicas, jurídicas y de todos los demás involucrados. Tampoco es algo trivial ni se puede tratar con frivolidad, pues debe haber claridad acerca de lo que es esencial. Una y otra vez los terremotos desnudan las debilidades de las prácticas constructivas (i.e. diseño, construcción, materiales, mante-nimiento) y de los sitios escogidos para realizarlas. Pero también, algunos terremotos recientes han subrayado las fortalezas, sobre todo y por ejemplo luego de los sismos que han sucedido entre 2007 y 2013 en Chile, Costa Rica, Guatemala, México, Colombia, Perú o aún mejor en el ejemplo de Christchurch, Nueva Zelanda (con magnitudes entre $M_{w} 6,5$ y 8,8), los cuales no generaron todos los daños posibles, comparados por ejemplo con el de Haití (12 de enero de $\left.2010, M_{w} 7,0\right)$, pues ya se cosechan los dividendos de haber comenzado a respetar códigos de construcción de buena calidad. Estas normas, junto con el ordenamiento territorial, seguirán siendo la mejor herramienta para mitigar el efecto de los terremotos y para salvaguardar la vida humana, las inversiones en infraestructura, las actividades productivas de bienes y servicios y la sostenibilidad del desarrollo. Hacia ello se deben seguir enfocando, siempre e incansablemente, los esfuerzos de la gestión del riesgo.

Es importante subrayar e insistir que la Sismología es una ciencia, que la gestión del riesgo derivado de la amenaza sísmica es un proceso de política pública y que ambas, para ser efectivas y justificar sus costos, deben armonizar su labor. Los científicos no deben difundir, por sí solos, información crítica que debería más bien vincularse con la seguridad pública. Un sismólogo no necesariamente tiene la habilidad para comunicarse con el público ni con los tomadores de decisiones. Una cosa es un pronóstico técnico, como los partes meteorológicos (IMN, 2014), o los informes que aparecen cotidianamente en las redes sociales acerca de los sismos, muchas veces apenas perceptibles por la población (RSN, 2014) y, otra muy diferente son las alertas, advertencias o alarmas relacionadas con un evento potencialmente destructivo. Estas últimas se deben dar a conocer, primero, a las autoridades y tomadores de decisiones competentes en el manejo del riesgo y de las emergencias y luego al público, con apoyo de especialistas en comunicación social. Esto es imprescindible si se trata de informar seria y responsablemente, lo cual no parece ser el caso cuando se intenta, a toda costa, insistir en tener la propiedad de la verdad absoluta y bajo el amparo de las libertades de expresión 
y de cátedra mal entendidas, sin haber medido de antemano todas las con-secuencias posibles (Pérez, 2012; Rojas, 2013). Asumir atribuciones de comunicador de manera unilateral y pretender informar con exceso o por omisión, sin mandato ni preparación profesional y, sobre todo, mediando la búsqueda oportunista de imagen personal sin importar las consecuencias, debería causar responsabilidad civil y penal.

Aunque la polémica sigue encendida acerca de la forma cómo la Fiscalía italiana ha interpretado la infor-mación, los estudios previos, el contenido de la Minuta, el Verbale y la conferencia de prensa luego de la reunión del 31 de marzo en L'Aquila, recientemente, la comunidad científica ha comenzado a reconocer que el verdadero alcance del juicio se relaciona más bien con la (in) capacidad y atribuciones de comunicación de los científicos, que con las falencias de la ciencia en sí misma, sus metodologías y lo que se espera de sus resultados (Ropeik, 2012). Fabio Picuti, el fiscal a cargo de la acusación indicó: “...This is not a trial against science; no-body can predict earthquakes [...] either they didn't know certain things, which is a problem, or they didn't know how to communicate what they did know, which is also a problem..." ("...este no es un juicio contra la ciencia; nadie puede predecir los sismos [...] o ellos no sabían ciertas cosas, lo cual es un problema, o no supieron comunicar lo que sabían, lo cual es también un problema" (Hall, 2011. Traducción del autor). Pero debe quedar claro que, para las poblaciones de los países expuestos a la amenaza sísmica, como por ejemplo en América Central, en donde los terremotos dañinos son frecuentes en casi cualquier momento y lugar, el pronóstico o la predicción de los terremotos solo contribuye a alimentar el estrés pre-sísmico y a fomentar la psicosis, y todo ello distrae la atención sobre otros asuntos de mayor prioridad, como por ejemplo la propia gestión del riesgo.

Ahora bien, sí es necesaria la "prognosis" de la amenaza sísmica, pero cuando es realizada profesionalmente, orientada hacia la evaluación de sus consecuencias, particularmente de los elementos expuestos y vulnerables y cuyo objetivo es el de mejorar su desempeño. El propósito debe ser la retroalimentación de los criterios para el di- seño estructural de las obras civiles, su ubicación segura, las prácticas constructivas correctas; además, para la generación de la conciencia pública y valoración de las ventajas y beneficios de la gestión del riesgo en general (Mora \& Alvarado; 2012a y 2012b).

Pero la prognosis misma debe difundirse sin lanzar alarmas innecesarias y a sabiendas de que se trabaja en un dominio de las ciencias en donde todavía están por resolverse muchas incertidumbres y en donde se debe comenzar a coordinar los esfuerzos entre las políticas públicas y la población, mediante la implantación de una disciplina operativa que incorpore claramente las prioridades y el papel de cada parte involucrada. Si los científicos prefieren, con prioridad, la búsqueda del lucro que les aporta su visibilidad mediática, la realidad es que no comprenden que laboran al servicio de la sociedad como fin último y, por lo tanto, han perdido los objetivos reales de su labor.

Por ello es que la sociedad difícilmente actuará en la dirección de la prevención, al menos hasta que se implanten las estrategias, planes y metodologías participativas, programas educativos y lineamientos políticos sostenibles, cuyo fin sea cambiar el paradigma de la negación, del aumento y de la amplificación irresponsable del riesgo. Es urgente, por lo tanto, que se establezca un balance para intervenir ese riesgo, introduciendo para ello los preceptos de la gestión del riesgo dentro de la cultura y que la sociedad escoja cuál repetición del escenario sísmico le parece mejor: Christchurch o Port-au-Prince...

\section{AGRADECIMIENTOS}

El autor agradece a Rosalba Barrios, Guillermo Alvarado, Omar Darío Cardona, Guillermo Alvarado, Vanessa Rosales, Percy Denyer, Walter Montero y Carolina Sigarán por sus aportes y sugerencias a este artículo.

\section{REFERENCIAS BIBLIOGRÁFICAS}

ALLEN, C., 1976: Responsibilities in earthquake prediction.- Bull. Seismol. Soc. Amer. 66(6): 2069-2074. 
AMORUSO, A. \& CRESCENTIN, L.; 2012: Preseismic phenomena from continuous nearfield strain meas-urements.- Bollettino di Geofisica Teorica ed Applicata, 53:169190.

BOSCHI, E., 2013: L'Aquila's Aftershocks Shake Scientists.- Science, 341, DOI:10.1126/ science.341.6153.1451

CAPORALE, G. \& DUSI, E., 2012: Il terremoto negato: All'Aquila possibili forti scosse.La Reppublica, 26 de octubre de 2012. http://inchieste.repubblica.it/it/repubblica/ rep-it/2012/10/26/news/allaquila possibili_scosse_fortissime_ecco_lallarme_censurato_da_bertolaso-45324109/ [Consulta: 10 de marzo de 2014].

FOX, S., 2009: Did a technician accurately forecast the L'Aquila earthquake, or was it a lucky guess? Scientific American, 7 de abril de 2009.- http://www.scientificamerican. com/article.cfm?id=radon-earthquake-prediction. [Consulta: 10 de marzo de 2014].

GELLER, R., JACKSON, D., KAGAN, \& MULARGIA, F., 1997: Earthquakes Cannot Be Predicted.- Science 275: 1616, DOI:10.1126/science.275.5306.1616.

GIULIANI, G., GIULIANI, R., TOTANI, G., EUSANI, G. \& TOTANI, F., 2009: Radon observations by Gamma Detectors "PM4 and PM-2" during the seismic period (January - April 2009) in L'Aquila Basin.Amer. Geophys. Union, Fall Meeting 2009, U14A-03.

GREENEMIEIR, L., 2011: Error and trial: Italian scientists face prison as earthquake manslaughter hearing resumes this weekend.Scientific American (30 de setiembre de 2011).- http://www.scientificamerican. com/article.cfm?id=italian-earthquakemanslaughter-hearing. [Consulta: $10 \mathrm{de}$ marzo de 2014].
GREENEMEIER, L., 2013: Faulty Justice: Italian Earthquake Scientist Speaks Out against His Conviction. Scientific American Sep. 26, 2013.- http://www.scientificamerican. com/article.cfm?id=italy-abruzzo-earthquake-scientist-trial. [Consulta: $10 \mathrm{de}$ marzo de 2014].

HALL, S., 2011: At Fault?.- Nature, 477: 264 269.

HOUGH, S., 2010: Predicting the Unpredictable: The Tumultuous Science of Earthquake Prediction.- 272 págs. Princeton University Press, Nueva York.

IMMÈ, G. \& MORELLI, D., 2012: Radon as Earthquake Precursor. Earthquake Research and Analysis. Dipartimento di Fisica e Astronomia Università di Catania - INFN Sezione di Catania, Italia.- http://cdn.intechopen.com/ pdfs/30707/InTech-Radon_as_earthquake_precursor.pdf. [Consulta: $10 \mathrm{de}$ marzo de 2014]

INSTITUTO NAZIONALE DI GEOFISICA E VULCANOLOGIA, 2003: Nuove norme sismiche - evoluzione, aggiornamenti, interventi.- http://www.protezionecivile.gov. it/jcms/it/classificazione.wp. [Consulta: 10 de marzo de 2014]

INSTITUTO NAZIONALE DI ASTROFISICA, 2009: Terremoto in Abruzzo. Comunicato INAF su Giuliani. Corriere della Sera (7 de abril de 2009).- http://forum.corriere.it/terremoto_nel_centro_italia/07-04-2009/comunicato_inaf_su giuliani-1235677.html. [Consulta: 10 de marzo de 2014]

INTERNATIONAL COMMISSION ON EARTHQUAKE FORECASTING FOR CIVIL PROTECTION, 2011: Operational Earthquake Forecasting: State of Knowledge and Guidelines for Utilization.An. Geohysics, 54(4): 315-391 
JACKSON, D., 1996: Hypothesis testing and earthquake prediction.- Proc. Nat. Acad. Sci. U.S.A. 93: 3772-3775.

MORA, S., 2009: Disasters are not natural. Risk management, a tool for development.Geol. Soc. London. Eng. Geol., Spec.Pub. 22: 101-112, DOI: 10.1144/EGSP22.7.

MORA, S., 2010: Disasters should not be the protagonists of Risk Management.- Geol. Active Proc. of the $11^{\text {th }}$ IAEG Congress. Auckland, New Zealand, 89-110.

MORA, S., 2012: La gestión del riesgo para enfrentar los mitos y realidades del calentamiento global antropogénico. Congreso argentino de Geología aplicada a la Ingeniería. Mendoza, Argentina.- Rev. Geol. Aplicada a la Ingeniería y al Ambiente, 28:111-125.

MORA, S. \& ALVARADO, G. 2012a: Enseñanzas del segundo sismo más grande de la historia costarricense. La Nación, Costa Rica.- http://www.nacion.com/foros/Ensenanzas-segundo-grande-historiacostarricense_0_1292670748.html. [Consulta: 10 de marzo de 2014]

MORA, S. \& ALVARADO, G., 2012b: Pronóstico sismológico versus construcción de buena calidad: Enseñan-zas del segundo sismo más grande de la historia costarricense.http://www.elpais.cr/frontend/noticia_detalle/3/72457. [Consulta: 10 de marzo de 2014].

MORENO, E., 2009: Terremoto en Italia: El "imbécil" que predijo el terremoto - Bajo mi sirena.- http://www.elenamoreno. net/2009/04/terremoto-italia-prediccion/. [Consulta: 10 de marzo de 2014].

MULARGIA, F. \& GASPERINI, P., 1992: Evaluating the statistical validity beyond chance of 'VAN' earth-quake precursors. Geophys. J. Int. 111: 32-44.
NOSENGO, N., 2013: Italian seismologists appeal L'Aquila ruling. Nature News.- http:// blogs.nature.com/news/2013/03/italianseismologists-appeal-laquila-ruling.html. [Consulta: 10 de marzo de 2014].

ORTOLANI, F. \& SPIZUOCO, A., 2010: Sisma dell'Aquila: responsabilità o penalizzazione della Commissione Grandi Rischi?.http://www.chiaianodiscarica.it/doc/sisma_grandi_rischi.pdf. [Consulta: $10 \mathrm{de}$ marzo de 2014].

PÉREZ, A., 2012: Jorge Marino Protti: No se vale criticar sin datos.- http://www.nacion. com/2012-09-16/RevistaDominical/JorgeMarino-Protti---No-se-vale-criticar-sindatos-.aspx? subsection=. [Consulta: $10 \mathrm{de}$ marzo de 2014].

PRITCHARD, C., 2012: L'Aquila ruling: Should scientists stop giving advice?.- http://www. bbc.co.uk/news/magazine-20097554.[Consulta: 10 de marzo de 2014]

PROTEZIONE CIVILE, 2003: Classificazione sismica d'Italia.- http://www.protezionecivile.gov.it/jcms/it/classificazione.wp. [Consulta: 10 de marzo de 2014].

PULINETS, S., OUZOUNOV, D., GIULIANI, G., CIRAOLO, L. \& TAYLOR, T.,. 2009: Atmosphere and radon activities observed prior to Abruzzo M6.3 earthquake of April 6, 2009.- Amer. Geophys. Union, Fall Meeting 2009, Abstract \#U14A-07.

ROJAS, P., 2013: Fuerte Terremoto ocurrirá dentro de 5 ó 10 años. http://www. crhoy.com/fuerte-terremoto-ocurriraen-osa-dentro-de-5-o-10-anos-segun-elovsicori/\#comment-84064. [Consulta: 10 de marzo de 2014].

ROPEIK, D., 2012: The L'Aquila verdict: A judgment not against science, but against a failure of science communication.- http:// 
blogs.scientificamerican.com/guestblog/2012/10/22/the-laquila-verdict-ajudgment-not-against-science-but-against-a-failure-of-science-communication/. [Consulta: 10 de marzo de 2014].

SCHOLZ, C., SYKES, L. \& AGGARWAL, Y., 1973: Earthquake Prediction. A Physical Basis. Science 181: 803-810. DOI:10.1126/science.181.4102.803.

STATI, D., 2009: Assessore Regionale de la Protezione Civile. Terremoto de L'Aquila, 31 marzo 2009. Youtu-be, 31 marzo 2009.- http://www.youtube.com/ watch? $v=8$ mienAqeWE8. [Consulta: 10 de marzo de 2014].

WYSS, M., 1997: Cannot Earthquakes Be Predicted?.- Science, 278: 487-490, DOI: 10.1126/science.278.5337.487.

\section{FUENTES HEMEROGRÁFICAS}

CODICE PENALE. ITALIA, 2013. Delle contravvenzioni di polizia.- http://www.altalex. com/index.php?idnot=36776 [Consulta: 10 de marzo de 2014].

COMMISSIONE GRANDI RISCHI., 2009: Verbale della comissionne Grandi Rischi.http://www.6aprile.it/docs/Protezione_ Civile/Verbali\%20commissione\%20grandi\%20rischi/commissione_grandi_rischi del_31_marzo_2009.pdf. [Consultas: 10 de marzo de 2014]:

CORRIERE DELLA SERA, 2013: Terremoto in Garfagnana: Panico per un tweet.- http:// www.corriere.it/cronache/13_gennaio_31/ allarme-provincia-di-lucca-evacuazionegarfagnana_f56903a0-6bf3-11e2-bfdf0d9d15b9395f.shtml. [Consulta: 10 de marzo de 2014].
DEPARTAMENTO DELLA PROTEZIONE CIVILE, 2009: Commissione nazionale per la previsione e prevenzione dei grandi rischi; rischio sísmico. (6 de abril de 2009).- http://www.ilmanifesto.it/fileadmin/archivi/PDF/verbale1.pdf [Consulta: 10 de marzo de 2014]

DICCIONARIO LAROUSSE, 2009: http://www. larousse.fr/dictionnaires/francais/pseudo science $/ 64764$ ?q=pseudoscience $\# 64038$. [Consulta: 10 de marzo de 2014].

EL PERIÓDICO, 2012: Los científicos claman contra la «ridícula» sentencia de L'Aquila.http://www.elperiodico.com/es/noticias/ sociedad/print-2232984.shtml. [Consulta: 10 de marzo de 2014].

EURONEWS, 2012: L'Aquila's Seven' to appeal manslaughter conviction.- http://www. euronews.com/2012/10/22/1-aquila-seven-to-appeal-manslaughter-conviction/. [Consulta: 10 de marzo de 2014].

GAZZETA DU SUD, 2012: Experts quit over L'Aquila quake verdict amid global shock.- http://www.gazzettadelsud.it/ news/english/19064/Experts-quit-overL-Aquila-quake-verdict-amid-globalshock.html [Consulta: 10 de marzo de 2014].

INSTITUTO METEOROLÓGICO NACIONAL DE COSTA RICA, 2014: Estado actual del tiempo y pronóstico por regiones y ciudades.- http://www.imn.ac.cr/. [Consulta: 10 de marzo de 2014].

KINGTON, T., 2012: In L'Aquila, Italy. The Guardian-The Observer, Saturday 27 October 2012.- http://www.guardian. co.uk/world/2012/oct/27/laquila-earthquake-battle-science-politics. [Consulta: 10 de marzo de 2014]. 
LABORATORI NAZIONALI DEL GRAN SASSO.- $\quad \mathrm{http} / / / w w w . l n g s . i n f n . i t /$. [Consulta: 10 de marzo de 2014].

LA NACIÓN, 2012: Seis años de prisión a científicos italianos por no predecir un terremoto. El Mundo.- http://www.lanacion. com.ar/1519667-seis-anos-de-prision-acientificos-italianos-por-no-predecir-unterremoto [Consulta: 10 de marzo de 2014]

LA REPPUBLICA, 2013: L'Aquila: crollo Casa dello Studente, condanne da 4 a 2 anni e mezzo. 16 de febrero de 2013. - http:// www.repubblica.it/cronaca/2013/02/16/ news/1_aquila_crollo_casa_dello_studente_condanne_da_4_a_2_anni_e_mezzo-52803213/

L'ESPRESSO, 2009: Verbale della commissione Grandi Rischi l'Aquila (31 de marzo 2009). - http://speciali.espresso.repubblica.it/pdf/terremoto/verbale.pdf [Consulta: 10 de marzo de 2014]

NATIONAL COMMISSION FOR FORECASTING AND PREVENTING MAJOR RISKS, 2009. - http://www.protezionecivile.gov.it/jcms/en/commissione grandi_rischi.wp?request locale $=$ en [Consulta: 10 de marzo de 2014]

DICCIONARIO DE LA LENGUA ESPAÑOLA (RAE), 22a Edición (2001).- http://rae.es/ drae. [Consulta: 10 de marzo de 2014].

\section{RED SISMOLÓGICA NACIONAL DE COSTA} RICA (RSN), 2013: ¿La ocurrencia de varios temblores pequeños puede evitar que suceda un gran terremoto?.- http:// www.rsn.ucr.ac.cr/index.php/es/preguntasfreceuntes/sismologia/331-la-ocurrenciade-muchos-temblores-pequenos-puedeevitar-que-ocurra-un-terremoto-grande. [Consulta: 10 de marzo de 2014].
RED SISMOLÓGICA NACIONAL DE COSTA RICA (RSN), 2014: Partes sismológicos y vulcanológicos.- http://www.rsn.ucr.ac.cr/. [Consulta: 10 de marzo de 2014].

RT-ACTUALIDAD, 2013: Científicos japoneses alertan de gigantesco terremoto "que está por venir".- http://actualidad.rt.com/ actualidad/view/95931-cientificos-japonfuerte-terremoto. [Consulta: 10 de marzo de 2014].

THE BIG PICTURE, 2009: The L'Aquila Earthquake Images. April 9, 2009.- http:// www.boston.com/bigpicture/2009/04/ comments_the_laquila_earthquake.html. [Consulta: 10 de marzo de 2014].

THE ECONOMIST, 2011: Scientists in the dock. 17 de setiembre de 2011.- http://www.economist.com/node/21529006. [Consulta: 10 de marzo de 2014].

TRIBUNALE DE L'AQUILA, 2012: Il giudice di l'Aquila. Nella pubblica udienza del $22 / 10 / 2012$ ha pronunciato la seguente sentenza.- http://processoaquila.files. wordpress.com/2013/01/sentenza-grandirischi-completa-1.pdf. [Consulta: $10 \mathrm{de}$ marzo de 2014].

UNITED STATES GEOLOGICAL SURVEYEarthquake Hazard Program-USGS-EHP: Magnitude 6.3 Cen-tral Italy. 2009 April 06 01:32:39 UTC.- http://earthquake. usgs.gov/earthquakes/eqinthenews/2009/ us2009fcaf/. [Consulta: 10 de marzo de 2014].

UNIVISIÓN, 2013: Pronostican sismo de 8.5 Richter en la Ciudad de México.- http://noticias.univision.com/article/1651430/2013-08-27/ mexico/noticias/pronostican-sismo-de-85-richter-en-la-ciudad-de-mexico. [Consulta: 10 de marzo de 2014]. 
